\title{
ANALYSIS OF A TIME OPTIMAL CONTROL PROBLEM RELATED TO THE MANAGEMENT OF A BIOREACTOR ${ }^{*, * *}$
}

\author{
Lino J. Alvarez-VÁzquez ${ }^{1}$, Francisco J. Fernández ${ }^{2}$ And Aurea Martínez ${ }^{1}$
}

\begin{abstract}
We consider a time optimal control problem arisen from the optimal management of a bioreactor devoted to the treatment of eutrophicated water. We formulate this realistic problem as a state-control constrained time optimal control problem. After analyzing the state system (a complex system of coupled partial differential equations with non-smooth coefficients for advection-diffusionreaction with Michaelis-Menten kinetics, modelling the eutrophication processes) we demonstrate the existence of, at least, an optimal solution. Then we present a detailed derivation of a first order optimality condition (involving the corresponding adjoint systems) characterizing these optimal solutions. Finally, a numerical example is shown.
\end{abstract}

Mathematics Subject Classification. 35D05, 49J20, 93C20.

Received February 10, 2009. Revised September 3, 2009 and January 21, 2010.

Published online April 23, 2010.

\section{Introduction: EnVIRONMENT AND MATHEMATICS}

An excessive concentration of nutrients (usually nitrogen or phosphorus) in large bodies of water (as lakes, estuaries, rivers and so on) encourages the growth of aquatic organisms, causing its aging by an abnormal biological enrichment of the water. For instance, in young lakes water is clear and cold, supporting a reduced quantity of life. However, urban sewage and agricultural/industrial wastes derived from human activities can introduce into the aquatic media a large amount of nutrients which promotes an increasing of the lake's fertility and accelerates the aging process: plant/animal life explodes, and organic detritus begin to be deposited on the bottom, giving raise to the eutrophication processes. The main pollutants (nitrates and phosphates) from drained wastewater act as plant nutrients, stimulating the algal blooms and, consequently, robbing the water of dissolved oxygen vital to other aquatic life species, and warming the water. These facts usually poison whole populations of fish, whose organic remains further exhaust the dissolved oxygen from water, leading the lake to ecological death.

\footnotetext{
Keywords and phrases. Time optimal control, partial differential equations, optimality conditions, existence, bioreactor.

* Supported by Project MTM2009-07749 of Micinn (Spain), and INCITE-09-291-083-PR of Xunta de Galicia.

** The authors thank the fruitful suggestions of Prof. Jean-Pierre Raymond. The authors also appreciate the interesting and detailed remarks of the anonymous referee.

1 Departamento de Matemática Aplicada II, E.T.S.I. Telecomunicación, Universidad de Vigo, 36310 Vigo, Spain.

lino@dma.uvigo.es; aurea@dma.uvigo.es

2 Departamento de Matemática Aplicada, Facultad de Matemáticas, Universidad de Santiago de Compostela, 15706 Santiago, Spain. fjavier.fernandez@usc.es
} 
Eutrophication of aquatic media has been considered one of the major threats to the health of ecosystems since the last decades. Eutrophication processes have been the subject of a wide range of biological/engineering researches but, from a purely mathematical viewpoint, related mathematical models have been much less analyzed. There exist several interesting papers studying the problem from a simplified ordinary differential equations viewpoint, but within the framework of partial differential equations, only a few models have been proposed: a 1D spatial model for oxygen dynamics was initially given by Lunardini and Di Cola [13]. More complex 2D depth-averaged models for nutrient-phytoplankton in shallow water have been given, for instance, by Arino et al. [5] or Cioffi and Gallerano [9]. For the full 3D case, several numerical models have been proposed, among others, by Drago et al. [10], Yamashiki et al. [20] or Park et al. [14], but only introducing numerical simulations for particular zones, without presenting theoretical results about existence or uniqueness of solution. The most remarkable results of existence of solution for a complete nutrient-phytoplankton-zooplankton-oxygen model can be found in the paper of Allegretto et al. [1] (for a particular periodic case) or in the recent work of the authors [3] (presenting also uniqueness and regularity results for a realistic situation with non-smooth water velocity).

In order to reduce this harmful concentration of nutrients in water, one of the most useful techniques is related to the use of bioreactors. The operation of this type of bioreactors consists of holding up eutrophicated water (rich, for instance, in nitrogen) in a small number of large tanks where we add a certain (small) quantity of phytoplankton, that we let grow in order to absorb nitrogen from water. In the particular problem analyzed in this work we consider only two large shallow tanks with the same capacities (but possibly with different geometries). Water rich in nitrogen will fill the first tank $\Omega_{1}$, where we will add a quantity $\rho^{1}$ of phytoplankton which we will let freely grow for a permanence time $T^{1}$ - to drop nitrogen level down to a desired threshold. Inside this first tank we are also interested in obtaining - with economical purposes - a certain quantity of organic detritus, since they are very estimated as agricultural fertilizers. Once reached the desired levels of nitrogen and organic detritus (settled in the bottom of the tank, then reclaimed for agricultural use), we will drain this first tank and pass water to the second tank $\Omega_{2}$, where the same operation is repeated, by adding a new amount $\rho^{2}$ of phytoplankton. Water leaving this second fermentation tank after a permanence time $T^{2}$ (thus, the total time for the whole process is $T^{1}+T^{2}$ ) will be usually poor in nitrogen, but rich in detritus (again settled in the bottom) and phytoplankton (recovered from a final filtering). At this point, we are also interested - for economical and ecological reasons - in minimizing this final quantity of phytoplankton. Thus, the time optimal control problem will consist of finding the quantities $\left(\rho^{1}, \rho^{2}\right)$ of phytoplankton that we must add to each one of both tanks along the time, and the minimal permanence times $\left(T^{1}, T^{2}\right)$, so that the nitrogen levels be lower that maximum thresholds and detritus levels be higher that minimum thresholds, and in such a way that the final phytoplankton concentration be as reduced as possible.

From a mathematical point of view, this problem can be formulated as a time optimal control problem with state and control constraints, where the control variable $\left(T^{1}, T^{2}, \rho^{1}, \rho^{2}\right)$ is the vector of permanence times and phytoplankton quantities added in each tank, the state variables are the concentrations of nutrient, phytoplankton, zooplankton and organic detritus, the cost function to be minimized is a combination of the permanence times and the phytoplankton concentration of water leaving the second tank, the state constraints stand for the thresholds required for the final nitrogen and detritus concentrations in each one of the tanks, and the control constraints are related to technological bounds.

The mathematical literature related to the analysis of time optimal control problems is not large. However, the number of references devoted to time optimal control of partial differential equations is more reduced. Among the most recent contributions on the topic we must mention those of Cannarsa and Frankowska [7] and Gugat and Leugering [11], where a semigroup theory approach is used, or those of Raymond $[4,15,16]$ and Wang $[12,18,19]$, dealing with academical related problems with the time as a control variable. In these papers very interesting results on the existence of solutions and optimality conditions are obtained. Unfortunately, none of them can be applied to our particular problem due to the lack of smoothness in the coefficients, and to their dependence on time. So, we will need to develop a complete analysis of the time optimal control problem specifically adapted to our non-smooth assumptions. 
A detailed mathematical formulation of the time optimal control problem is presented in Section 2. The next section is devoted to the analysis of the state system, and to the demonstration of the existence of a (non necessarily unique) optimal solution. Finally, these optimal solutions will be characterized by a first order optimality condition (involving a suitable adjoint system to be adequately defined) presented in Section 4 . We will also introduce an alternative formulation of the optimality conditions for the qualified case.

\section{Formulation of the time optimal Control problem}

Most realistic mathematical models governing eutrophication are recently formulated as systems of partial differential equations (as opposite to classical systems of ordinary differential equations) with a high complexity due to the great variety of internal phenomena appearing on them. In this work we have considered a complete model, where the four biological variables involved in our problem appear (the formulation of the biochemical interaction terms and their meaning can be found, for instance, in Canale [6]). Thus, we consider the state variable $\mathbf{u}=\left(u^{1}, u^{2}, u^{3}, u^{4}\right)$, where $u^{1}$ denotes a generic nutrient concentration, for instance, nitrogen (as will be considered in our case) or phosphorus, $u^{2}$ denotes the phytoplankton concentration, $u^{3}$ denotes the zooplankton concentration, and $u^{4}$ denotes the organic detritus concentration.

The interaction of these four biological species into a given water domain $\Omega \subset \mathbb{R}^{3}$ (with a smooth enough boundary $\partial \Omega$ ) and along a time interval $I=(0, T)$ can be described by the following system of coupled partial differential equations for convection-diffusion-reaction with standard Michaelis-Menten kinetics:

$$
\begin{cases}\frac{\partial u^{1}}{\partial t}+\mathbf{w} \cdot \nabla u^{1}-\nabla \cdot\left(\mu_{1} \nabla u^{1}\right)+C_{n c} L \frac{u^{1}}{K_{N}+u^{1}} u^{2}-C_{n c} K_{r} u^{2}-C_{n c} K_{r d} \Theta^{\theta-20} u^{4}=g^{1} & \text { in } Q, \\ \frac{\partial u^{2}}{\partial t}+\mathbf{w} \cdot \nabla u^{2}-\nabla \cdot\left(\mu_{2} \nabla u^{2}\right)-L_{\frac{u^{1}}{K_{N}+u^{1}}} u^{2}+K_{r} u^{2}+K_{m f} u^{2}+K_{z} \frac{u^{2}}{K_{F}+u^{2}} u^{3}=g^{2} & \text { in } Q, \\ \frac{\partial u^{3}}{\partial t}+\mathbf{w} \cdot \nabla u^{3}-\nabla \cdot\left(\mu_{3} \nabla u^{3}\right)-C_{f z} K_{z} \frac{u^{2}}{K_{F}+u^{2}} u^{3}+K_{m z} u^{3}=g^{3} & \text { in } Q, \\ \frac{\partial u^{4}}{\partial t}+\mathbf{w} \cdot \nabla u^{4}-\nabla \cdot\left(\mu_{4} \nabla u^{4}\right)-K_{m f} u^{2}-K_{m z} u^{3}+K_{r d} \Theta^{\theta-20} u^{4}+W_{f d} \frac{\partial u^{4}}{\partial x_{3}}=g^{4} & \text { in } Q,\end{cases}
$$

with corresponding boundary conditions on $\Sigma$ and initial conditions in $\Omega$, and where we have considered:

- $Q=I \times \Omega, \quad \Sigma=I \times \partial \Omega$;

- $\mathbf{w}(t, x)$ : the water velocity;

- $\mu_{i}, i=1, \ldots, 4$ : the diffusion coefficients of each species, which are positive constants;

- $C_{n c}$ : the nitrogen-carbon stoichiometric relation;

- $L(t, x)$ : the luminosity function, given by expression

$$
L(t, x)=\nu C_{t}^{\theta(t, x)-20} \frac{I_{0}}{I_{s}} \mathrm{e}^{-\phi x_{3}}
$$

with $\nu$ the maximum phytoplankton growth rate, $C_{t}$ the phytoplankton growth thermic constant, $\theta(t, x)$ the water temperature (in Celsius), $I_{0}$ the incident light intensity, $I_{s}$ the light saturation, and $\phi$ the light absorption by water;

- $K_{N}$ : the nitrogen half-saturation constant;

- $K_{r d}$ : the detritus regeneration rate;

- $\Theta$ : the detritus regeneration thermic constant, which is positive;

- $K_{r}$ : the phytoplankton endogenous respiration rate;

- $K_{m f}$ : the phytoplankton death rate;

- $K_{z}$ : the zooplankton predation (grazing);

- $K_{F}$ : the phytoplankton half-saturation constant;

- $C_{f z}$ : the grazing efficiency factor;

- $K_{m z}$ : the zooplankton death rate (including predation);

- $W_{f d}$ : the falling velocity of organic detritus (settling). 
In order to present in a simple way the system of equations (2.1) we will consider, for $\mathbb{R}^{+}=[0, \infty)$, the mapping $\mathbf{A}=\left(A^{1}, A^{2}, A^{3}, A^{4}\right): \mathbb{R}^{+} \times \Omega \times\left[\mathbb{R}^{+}\right]^{4} \longrightarrow \mathbb{R}^{4}$, given by:

$$
\mathbf{A}(t, x, \mathbf{u})=\left[\begin{array}{c}
-C_{n c}\left[L(t, x) \frac{u^{1}}{K_{N}+u^{1}} u^{2}-K_{r} u^{2}\right]+C_{n c} K_{r d} \Theta^{\theta(t, x)-20} u^{4} \\
{\left[L(t, x) \frac{u^{1}}{K_{N}+u^{1}} u^{2}-K_{r} u^{2}\right]-K_{m f} u^{2}-K_{z} \frac{u^{2}}{K_{F}+u^{2}} u^{3}} \\
C_{f z} K_{z} \frac{u^{2}}{K_{F}+u^{2}} u^{3}-K_{m z} u^{3} \\
K_{m f} u^{2}+K_{m z} u^{3}-K_{r d} \Theta^{\theta(t, x)-20} u^{4}
\end{array}\right] .
$$

Moreover, the sedimentation term $W_{f d} \frac{\partial u^{4}}{\partial x_{3}}$ in the fourth equation of (2.1) will be incorporated into the convective term $\mathbf{w} \cdot \nabla u^{4}$, by redefining a new artificial velocity $\mathbf{w}+\left(0,0, W_{f d}\right)$ for the organic detritus equation. Thus, taking into account above considerations, the system (2.1) can be written in the following equivalent way:

$$
\frac{\partial u^{i}}{\partial t}+\mathbf{w}_{i} \cdot \nabla u^{i}-\nabla \cdot\left(\mu_{i} \nabla u^{i}\right)=A^{i}(t, x, \mathbf{u})+g^{i} \quad \text { in } Q, \quad \text { for } i=1, \ldots, 4,
$$

where $\mathbf{w}_{i}=\mathbf{w}$ for $i=1,2,3$, and $\mathbf{w}_{4}=\mathbf{w}+\left(0,0, W_{f d}\right)$.

With these notations we can mathematically formulate the time optimal control problem with the following elements:

- Controls: As we have remarked in the Introduction, we will control the system by means of four design variables: the permanence time $T^{1}$ of water inside the first tank $\Omega_{1}$, the permanence time $T^{2}$ inside the second tank $\Omega_{2}$, the quantity $\rho^{1}(t, x)$ of phytoplankton added in the first tank along the time interval $I_{1}=\left(0, T^{1}\right)$, and the quantity $\rho^{2}(t, x)$ of phytoplankton added in the second tank along the corresponding time interval $I_{2}=\left(0, T^{2}\right)$.

It is worthwhile remarking here that, although the time-space variables are different for each tank $\left(\left(t_{1}, x_{1}\right) \in I_{1} \times \Omega_{1}\right.$ for the first tank, $\left(t_{2}, x_{2}\right) \in I_{2} \times \Omega_{2}$ for the second one), for the sake of simplicity, we will use the same variables $(t, x)$ for both cases, since no confusion is possible.

- State systems: We consider two state systems giving the concentrations of nitrogen, phytoplankton, zooplankton, and organic detritus in each one of the tanks. Since both tanks are isolated, no transference for any of the four species is considered across their boundaries, that is, Neumann boundary conditions are assumed to be null for all concentrations in both tanks. In addition, both systems are coupled by means of the initial-final conditions: when water passes from the first tank to the second one, it is natural to assume that water is mixed up, and this is the reason of considering the initial conditions for the concentrations inside the second tank as given by the corresponding averaged final concentrations in the first tank. So, the two coupled state systems are given by:

- Tank $\Omega_{1}$ : The state variables for the first tank will be denoted $\mathbf{u}^{1}=\left(u^{1,1}, u^{2,1}, u^{3,1}, u^{4,1}\right)$ with $u^{1,1}$ (nitrogen), $u^{2,1}$ (phytoplankton), $u^{3,1}$ (zooplankton), and $u^{4,1}$ (organic detritus). The permanence time of water inside this first tank will be $T^{1}$, the fluid velocity in the tank will be $\mathbf{w}^{1}(t, x)$, and the initial concentrations will be given by $\mathbf{u}_{0}^{1}=\left(u_{0}^{1,1}, u_{0}^{2,1}, u_{0}^{3,1}, u_{0}^{4,1}\right)$. Thus, for $Q_{1}=I_{1} \times \Omega_{1}$ and $\Sigma_{1}=I_{1} \times \partial \Omega_{1}$, we have the system, for $i=1, \ldots, 4$ :

$$
\begin{cases}\frac{\partial u^{i, 1}}{\partial t}+\mathbf{w}_{i}^{1} \cdot \nabla u^{i, 1}-\nabla \cdot\left(\mu_{i} \nabla u^{i, 1}\right)=A^{i}\left(t, x, \mathbf{u}^{1}\right)+ \begin{cases}\rho^{1}, & \text { if } i=2 \\ 0, & \text { if } i \neq 2\end{cases} & \text { in } Q_{1}, \\ \frac{\partial u^{i, 1}}{\partial n}=0 & \text { on } \Sigma_{1}, \\ u^{i, 1}(0)=u_{0}^{i, 1} & \text { in } \Omega_{1} .\end{cases}
$$

- Tank $\Omega_{2}$ : The state variables for the second tank will be denoted $\mathbf{u}^{2}=\left(u^{1,2}, u^{2,2}, u^{3,2}, u^{4,2}\right)$ with $u^{1,2}$ (nitrogen), $u^{2,2}$ (phytoplankton), $u^{3,2}$ (zooplankton), and $u^{4,2}$ (organic detritus). The permanence time of water inside this second tank will be $T^{2}$, and the fluid velocity in the tank will be 
$\mathbf{w}^{2}(t, x)$. Thus, for $Q_{2}=I_{2} \times \Omega_{2}$ and $\Sigma_{2}=I_{2} \times \partial \Omega_{2}$, we have the analogous system, for $i=1, \ldots, 4$ :

$$
\left\{\begin{array}{lll}
\frac{\partial u^{i, 2}}{\partial t}+\mathbf{w}_{i}^{2} \cdot \nabla u^{i, 2}-\nabla \cdot\left(\mu_{i} \nabla u^{i, 2}\right)=A^{i}\left(t, x, \mathbf{u}^{2}\right)+ \begin{cases}\rho^{2}, & \text { if } i=2 \\
0, & \text { if } i \neq 2\end{cases} & \text { in } Q_{2}, \\
\frac{\partial u^{i, 2}}{\partial n}=0 & & \text { on } \Sigma_{2}, \\
u^{i, 2}(0)=\frac{1}{\operatorname{meas}\left(\Omega_{1}\right)} M_{1}^{i}\left(\mathbf{u}^{1}\left(T^{1}\right)\right) & \text { in } \Omega_{2},
\end{array}\right.
$$

where $\mathbf{M}_{j}=\left(M_{j}^{1}, M_{j}^{2}, M_{j}^{3}, M_{j}^{4}\right)$, for $j=1,2$, are the functionals, defined from $\left[L^{1}\left(\Omega_{j}\right)\right]^{4}$ to $\mathbb{R}^{4}$, given by:

$$
\mathbf{M}_{j}\left(\mathbf{v}^{j}\right)=\left[\begin{array}{c}
\int_{\Omega_{j}} v^{1, j} \mathrm{~d} x \\
\int_{\Omega_{j}} v^{2, j} \mathrm{~d} x \\
\int_{\Omega_{j}} v^{3, j} \mathrm{~d} x \\
0
\end{array}\right]
$$

We have to note here that, since detritus settle before water passes to the second tank, the initial organic detritus concentration $u^{4,2}(0)$ (that is, the fourth component of $\mathbf{M}_{1}\left(\mathbf{u}^{1}\left(T^{1}\right)\right)$ ) will be considered null.

- Objective function: Due to the fact that we are interested in reducing the total processing time $T^{1}+T^{2}$ of water inside the bioreactor, and in minimizing the final phytoplankton concentration of water leaving the second tank, we are led to consider the following cost functional $J$ given by:

$$
J\left(T^{1}, T^{2}, \rho^{1}, \rho^{2}\right)=N_{1}\left(T^{1}+T^{2}\right)+\frac{N_{2}}{\operatorname{meas}\left(\Omega_{2}\right)} \int_{\Omega_{2}} u^{2,2}\left(T^{2}\right) \mathrm{d} x
$$

where $N_{1}, N_{2} \geq 0$ are two scaling factors.

- State constraints: As commented in the first section, the final nitrogen concentration in each tank must be lower than a given threshold, and the final organic detritus concentration in each tank must be greater than another given threshold. These constraints translate into the relations given by $\mathbf{B}=\left(B^{1}, B^{2}, B^{3}, B^{4}\right)$ :

$$
\left\{\begin{array}{l}
B^{1}\left(T^{1}, T^{2}, \rho^{1}, \rho^{2}\right)=\frac{1}{\operatorname{meas}\left(\Omega_{1}\right)} \int_{\Omega_{1}} u^{1,1}\left(T^{1}\right) \mathrm{d} x \leq \sigma_{1}, \\
B^{2}\left(T^{1}, T^{2}, \rho^{1}, \rho^{2}\right)=\frac{1}{\operatorname{meas}\left(\Omega_{2}\right)} \int_{\Omega_{2}} u^{1,2}\left(T^{2}\right) \mathrm{d} x \leq \sigma_{2}, \\
B^{3}\left(T^{1}, T^{2}, \rho^{1}, \rho^{2}\right)=\frac{1}{\operatorname{meas}\left(\Omega_{1}\right)} \int_{\Omega_{1}} u^{4,1}\left(T^{1}\right) \mathrm{d} x \geq \theta_{1}, \\
B^{4}\left(T^{1}, T^{2}, \rho^{1}, \rho^{2}\right)=\frac{1}{\operatorname{meas}\left(\Omega_{2}\right)} \int_{\Omega_{2}} u^{4,2}\left(T^{2}\right) \mathrm{d} x \geq \theta_{2},
\end{array}\right.
$$

for certain given values $\sigma_{1}, \sigma_{2}, \theta_{1}, \theta_{2}>0$.

- Control constraints: Finally, for technological reasons, the permanence times $\left(T^{1}, T^{2}\right)$ must take their values between two fixed bounds $0<T_{\min }<T_{\max }<\infty$, that is, they must lie in the set

$$
\mathcal{V}_{a d}^{1}=\left\{\left(T^{1}, T^{2}\right) \in \mathbb{R}^{2}: T_{\min } \leq T^{1}, T^{2} \leq T_{\max }\right\}
$$

which is a compact, convex, and nonempty subset of $\mathbb{R}^{2}$. 
By similar motivations, the quantities $\left(\rho^{1}, \rho^{2}\right)$ of phytoplankton added to the tanks must be nonnegative and bounded by a maximal admissible value $K>0$, that is, they must lie in the set

$$
\begin{aligned}
\mathcal{V}_{a d}^{2}= & \left\{\left(\rho^{1}, \rho^{2}\right) \in L^{2}\left(\left(0, T_{\max }\right) \times \Omega_{1}\right) \times L^{2}\left(\left(0, T_{\max }\right) \times \Omega_{2}\right):\right. \\
& 0 \leq \rho^{1}(t, x) \leq K \text { a.e. }(t, x) \in\left(0, T_{\max }\right) \times \Omega_{1}, \\
& \left.0 \leq \rho^{2}(t, x) \leq K \text { a.e. }(t, x) \in\left(0, T_{\max }\right) \times \Omega_{2}\right\}
\end{aligned}
$$

which is a closed, bounded, convex, and nonempty subset of $L^{2}\left(\left(0, T_{\max }\right) \times \Omega_{1}\right) \times L^{2}\left(\left(0, T_{\max }\right) \times \Omega_{2}\right)$.

In order to simplify the notations, we will denote $\mathcal{V}_{a d}=\mathcal{V}_{a d}^{1} \times \mathcal{V}_{a d}^{2}$. So, the control must satisfy $\left(T^{1}, T^{2}, \rho^{1}, \rho^{2}\right) \in \mathcal{V}_{a d}$.

Thus, the formulation of the time optimal control problem, denoted by $(\mathcal{P})$, will be the following:

$(\mathcal{P}) \quad \inf \left\{J\left(T^{1}, T^{2}, \rho^{1}, \rho^{2}\right)\right.$ such that $\left(T^{1}, T^{2}, \rho^{1}, \rho^{2}\right) \in \mathcal{V}_{a d}$ and $\left(\mathbf{u}^{1}, \mathbf{u}^{2}\right)$ satisfies $(2.4)-(2.5)$ and $\left.(2.8)\right\}$.

\section{Analysis of the CONTROL PROBlem}

Since the problem we are dealing with depends on varying (although bounded) final times, we need to assume that the water velocities $\mathbf{w}^{j}, j=1,2$, and the corresponding water temperatures $\theta^{j}, j=1,2$, in the coupled state systems (2.4)-(2.5) are defined all along the whole time interval $\left[0, T_{\max }\right]$.

As a first step we will introduce several notations for the functional spaces to be used in the below sections: We denote $L_{\sigma}^{3}\left(\Omega_{j}\right)$ the closure in $L^{3}\left(\Omega_{j}\right)^{3}$ of the space $\left\{\mathbf{v} \in \mathcal{D}\left(\Omega_{j}\right)^{3}: \operatorname{div}(\mathbf{v})=0\right\}, j=1,2$. We define $\mathbf{V}_{j}=\left[H^{1}\left(\Omega_{j}\right)\right]^{4}, \mathbf{H}_{j}=\left[L^{2}\left(\Omega_{j}\right)\right]^{4}, j=1,2$, which can be considered within the classical framework $\mathbf{V}_{j} \subset \mathbf{H}_{j} \equiv$ $\mathbf{H}_{j}^{\prime} \subset \mathbf{V}_{j}, j=1,2$. Finally, $W^{1, p, q}\left(0, T_{\max } ; V, V^{\prime}\right)$ denotes the functional space given by:

$$
W^{1, p, q}\left(0, T_{\max } ; V, W\right)=\left\{v \in L^{p}\left(0, T_{\max } ; V\right): \frac{\mathrm{d} v}{\mathrm{~d} t} \in L^{q}\left(0, T_{\max } ; W\right)\right\}
$$

for any Banach spaces $V \subset W$, and for $1 \leq p, q \leq \infty$.

Then, as was demonstrated by the authors in [3] (using similar arguments to previous paper [2] for a problem related to food technology), the eutrophication systems (2.4)-(2.5) admit a solution under non-smooth hypotheses. To be exact, if we assume that the initial conditions and the source terms are nonnegative and bounded, and that the fluid velocities and temperatures satisfy:

$$
\begin{aligned}
& \mathbf{w}^{j} \in L^{2+\delta}\left(0, T_{\max } ; L_{\sigma}^{3}(\Omega)\right), \quad j=1,2, \quad \text { for some } \delta \geq 0, \\
& \theta^{j} \in L^{2}\left(\left(0, T_{\max }\right) \times \Omega_{j}\right), \quad j=1,2, \\
& \quad \text { such that } 0 \leq \theta_{j}(t, x) \leq M \text { a.e. }(t, x) \in\left(0, T_{\max }\right) \times \Omega_{j},
\end{aligned}
$$

then the state systems $(2.4)-(2.5)$ admit a unique solution $\mathbf{u}^{j} \in W^{1,2,1+\epsilon}\left(0, T_{\max } ; \mathbf{V}_{j}, \mathbf{V}_{j}^{\prime}\right) \cap\left[L^{\infty}\left(\left(0, T_{\max }\right) \times \Omega_{j}\right)\right]^{4}$, $j=1,2$, for a certain small $\epsilon \geq 0$. (In particular, for $\delta=0$ we have $\epsilon=0$, and for $\delta \rightarrow \infty$, we have $\epsilon \rightarrow 1$; see [3].) Moreover, this solution $\left(\mathbf{u}^{1}, \mathbf{u}^{2}\right)$ is nonnegative and bounded (in previous space norm) by a value depending on the time bound $T_{\max }$, the fluid velocities $\left(\mathbf{w}^{1}, \mathbf{w}^{2}\right)$, the controls $\left(\rho^{1}, \rho^{2}\right)$, and the initial condition $\mathbf{u}_{0}^{1}$.

Unfortunately these mild hypotheses - which will assure the existence of solution for the time optimal control problem $(\mathcal{P})$ - are not enough in order to allow us the derivation of optimality conditions for this problem. To do this we will need to impose the following stronger hypotheses on water velocities and temperatures:

$$
\begin{aligned}
& \mathbf{w}^{j} \in W^{1, \infty, \infty}\left(0, T_{\max } ; L_{\sigma}^{3}\left(\Omega_{j}\right), L_{\sigma}^{3}\left(\Omega_{j}\right)\right), \quad j=1,2, \\
& \theta^{j} \in W^{1, \infty, \infty}\left(0, T_{\max } ; L^{\infty}\left(\Omega_{j}\right), L^{\infty}\left(\Omega_{j}\right)\right), \quad j=1,2, \\
& \quad \text { such that } 0 \leq \theta_{j}(t, x) \leq M \text { a.e. }(t, x) \in\left(0, T_{\max }\right) \times \Omega_{j} .
\end{aligned}
$$


Finally, we will introduce the following definition:

Definition 3.1. We will say that $\left(T^{1}, T^{2}, \rho^{1}, \rho^{2}\right) \in \mathcal{V}_{a d}$ is a feasible control for the time optimal control problem $(\mathcal{P})$ if the associated state $\left(\mathbf{u}^{1}, \mathbf{u}^{2}\right)$, solution of coupled systems (2.4)-(2.5), satisfies the constraints (2.8).

Then, we can prove the following existence result under mild hypotheses (3.1):

Theorem 3.2. Let us assume that the set of feasible controls is nonempty. Let $\mathbf{u}_{0}^{1} \in\left[L^{\infty}\left(\Omega_{1}\right)\right]^{4}$ be such that $0 \leq u_{0}^{i, 1}(x) \leq M$ a.e. $x \in \Omega_{1}, i=1, \ldots, 4$, and let us assume that the water velocities and temperatures $\left(\mathbf{w}^{1}, \mathbf{w}^{2}, \theta^{1}, \theta^{2}\right)$ satisfy the regularity hypotheses (3.1), for $\delta>0$. Then, there exist elements $\left(\widetilde{T}^{1}, \widetilde{T}^{2}, \widetilde{\rho}^{1}, \widetilde{\rho}^{2}, \widetilde{\mathbf{u}}^{1}, \widetilde{\mathbf{u}}^{2}\right)$ $\in \mathcal{V}_{a d} \times\left(W^{1,2,1+\epsilon}\left(0, \widetilde{T}^{1} ; \mathbf{V}_{1}, \mathbf{V}_{1}^{\prime}\right) \cap\left[L^{\infty}\left(\left(0, \widetilde{T}^{1}\right) \times \Omega_{1}\right)\right]^{4}\right) \times\left(W^{1,2,1+\epsilon}\left(0, \widetilde{T}^{2} ; \mathbf{V}_{2}, \mathbf{V}_{2}^{\prime}\right) \cap\left[L^{\infty}\left(\left(0, \widetilde{T}^{2}\right) \times \Omega_{2}\right)\right]^{4}\right)$, with $\epsilon>0$, such that $\left(\widetilde{T}^{1}, \widetilde{T}^{2}, \widetilde{\rho}^{1}, \widetilde{\rho}^{2}\right)$ is a solution of the time optimal control problem $(\mathcal{P})$ with associated state $\left(\widetilde{\mathbf{u}}^{1}, \widetilde{\mathbf{u}}^{2}\right)$.

Proof. To prove the existence of optimal control for problem $(\mathcal{P})$ we will consider a minimizing sequence $\left\{\left(T_{n}^{1}, T_{n}^{2}, \rho_{n}^{1}, \rho_{n}^{2}, \mathbf{u}_{n}^{1}, \mathbf{u}_{n}^{2}\right)\right\}_{n \in \mathbb{N}} \subset \mathcal{V}_{a d} \times\left(W^{1,2,1+\epsilon}\left(0, T_{n}^{1} ; \mathbf{V}_{1}, \mathbf{V}_{1}^{\prime}\right) \cap\left[L^{\infty}\left(\left(0, T_{n}^{1}\right) \times \Omega_{1}\right)\right]^{4}\right) \times\left(W^{1,2,1+\epsilon}\left(0, T_{n}^{2} ; \mathbf{V}_{2}, \mathbf{V}_{2}^{\prime}\right) \cap\right.$ $\left.\left[L^{\infty}\left(\left(0, T_{n}^{2}\right) \times \Omega_{2}\right)\right]^{4}\right)$, that is, for all $n \in \mathbb{N}$, the pair $\left(\mathbf{u}_{n}^{1}, \mathbf{u}_{n}^{2}\right)$ verifies the state systems (2.4)-(2.5) with associated control $\left(\rho_{n}^{1}, \rho_{n}^{2}\right)$, satisfies the constraints (2.8), and:

$$
\begin{array}{r}
\lim _{n \rightarrow \infty} J\left(T_{n}^{1}, T_{n}^{2}, \rho_{n}^{1}, \rho_{n}^{2}\right)=\inf \left\{J\left(T^{1}, T^{2}, \rho^{1}, \rho^{2}\right) \text { such that }\left(T^{1}, T^{2}, \rho^{1}, \rho^{2}\right) \in \mathcal{V}_{a d}\right. \\
\left.\quad \text { and }\left(\mathbf{u}^{1}, \mathbf{u}^{2}\right) \text { satisfies }(2.4)-(2.5) \text { and }(2.8)\right\} .
\end{array}
$$

From the uniqueness of solution of state systems (2.4)-(2.5) we can assure that $\left(\mathbf{u}_{n}^{1}, \mathbf{u}_{n}^{2}\right)$ are defined for the whole time interval $\left(0, T_{\max }\right)$, for all $n \in \mathbb{N}$. Then we have, thanks to the estimates obtained in [3] and to the boundedness of the set $\mathcal{V}_{a d}$, that the sequence of states $\left\{\left(\mathbf{u}_{n}^{1}, \mathbf{u}_{n}^{2}\right)\right\}_{n \in \mathbb{N}}$ is bounded, i.e.

$$
\left.\left\|\mathbf{u}_{n}^{j}\right\|_{W^{1,2,1+\epsilon}\left(0, T_{\max }\right.} ; \mathbf{V}_{j}, \mathbf{V}_{j}^{\prime}\right) \cap\left[L^{\infty}\left(\left(0, T_{\max }\right) \times \Omega_{j}\right)\right]^{4} \leq C\left(T_{\max }, M\right), \quad j=1,2, \quad \forall n \in \mathbb{N} .
$$

Thus, since the inclusion $W^{1,2,1+\epsilon}\left(0, T_{\max } ; \mathbf{V}_{j}, \mathbf{V}_{j}^{\prime}\right) \cap\left[L^{\infty}\left(\left(0, T_{\max }\right) \times \Omega_{j}\right)\right]^{4} \subset\left[L^{\frac{10}{3}-\gamma}\left(\left(0, T_{\max }\right) \times \Omega_{j}\right)\right]^{4}$ is compact $\forall \gamma>0$ (for instance, applying Lem. 7.8 of Roubíček [17] with the choices $V_{1}=H^{1}\left(\Omega_{j}\right), V_{2}=L^{6-\delta}\left(\Omega_{j}\right)$ (for any $\delta>0$ small we have that the inclusion $H^{1}\left(\Omega_{j}\right) \subset L^{6-\delta}\left(\Omega_{j}\right)$ is compact), $V_{3}=H^{1}\left(\Omega_{j}\right)^{\prime}, H=L^{2}\left(\Omega_{j}\right)$ and $V_{4}=$ $L^{\mu}\left(\Omega_{j}\right)$ (with any $\mu^{-1}=\frac{1-\lambda}{2}+\frac{\lambda}{6-\delta}$ for $\lambda \in(0,1)$ ), we have the compact inclusion $W^{1,2,1+\epsilon}\left(0, T_{\max } ; \mathbf{V}_{j}, \mathbf{V}_{j}^{\prime}\right) \cap$ $L^{\infty}\left(\left(0, T_{\max }\right) \times \Omega_{j}\right) \subset L^{\frac{2}{\lambda}}\left(0, T_{\max } ; L^{\mu}\left(\Omega_{j}\right)\right)$; then, by choosing $\delta>0$ such that $\mu=\frac{2}{\lambda}=\frac{10}{3}-\gamma$ we obtain the desired inclusion (we must recall here that $p=\frac{10}{3}$ is the greatest exponent $p$ such that $L^{2}\left(0, T_{\max } ; H^{1}\left(\Omega_{j}\right)\right) \cap$ $\left.L^{\infty}\left(0, T_{\max } ; L^{2}\left(\Omega_{j}\right)\right) \subset L^{p}\left(\left(0, T_{\max }\right) \times \Omega_{j}\right)\right)$, there exist subsequences, still denoted in the same way, such that:

$$
\begin{array}{rll}
\rho_{n}^{j} & \rightarrow \widetilde{\rho}^{j} & \text { in } L^{2}\left(\left(0, T_{\max }\right) \times \Omega_{j}\right), \quad j=1,2, \\
\mathbf{u}_{n}^{j} & \rightarrow \widetilde{\mathbf{u}}^{j} & \text { in } L^{2}\left(0, T_{\max } ; \mathbf{V}_{j}\right), \quad j=1,2, \\
\mathbf{u}_{n}^{j} & \rightarrow \widetilde{\mathbf{u}}^{j} & \text { in }\left[L^{\frac{10}{3}}-\gamma\left(\left(0, T_{\max }\right) \times \Omega_{j}\right)\right]^{4}, \quad \forall \gamma>0, \quad j=1,2, \\
\mathbf{u}_{n}^{j}(t) & \rightarrow \widetilde{\mathbf{u}}^{j}(t) & \text { in } \mathbf{H}_{j}, \quad \forall t \in\left[0, T_{\max }\right], \quad j=1,2, \\
T_{n}^{j} & \rightarrow \widetilde{T}^{j} & \text { in } \mathbb{R}, \quad j=1,2 .
\end{array}
$$

As a first point we will demonstrate that $\left(\widetilde{\mathbf{u}}^{1}, \widetilde{\mathbf{u}}^{2}\right)$ is the state associated to the control $\left(\widetilde{T}^{1}, \widetilde{T}^{2}, \widetilde{\rho}^{1}, \widetilde{\rho}^{2}\right)$. In order to do this, we will pass to the limit in the state systems satisfied by the states $\left(\mathbf{u}_{n}^{1}, \mathbf{u}_{n}^{2}\right)$ associated to the controls $\left(T_{n}^{1}, T_{n}^{2}, \rho_{n}^{1}, \rho_{n}^{2}\right)$. We consider the very weak formulation of these state systems as defined 
in $\left(0, T_{\max }\right) \times \Omega_{1}$ and $\left(0, T_{\max }\right) \times \Omega_{2}$, respectively:

$$
\begin{gathered}
\sum_{i=1}^{4}\left\{\int_{\left(0, T_{\max }\right) \times \Omega_{1}} \mu_{i} \nabla u_{n}^{i, 1} \cdot \nabla z^{i} \mathrm{~d} x \mathrm{~d} t+\int_{\left(0, T_{\max }\right) \times \Omega_{1}} \mathbf{w}_{i}^{1} \cdot \nabla u_{n}^{i, 1} z^{i} \mathrm{~d} x \mathrm{~d} t-\int_{0}^{T_{\max }}\left\langle\frac{\mathrm{d} z^{i}}{\mathrm{~d} t}(t), u_{n}^{i, 1}(t)\right\rangle_{\mathbf{V}_{1}^{\prime}, \mathbf{V}_{1}} \mathrm{~d} t\right\} \\
=\sum_{i=1}^{4}\left\{\int_{\left(0, T_{\max }\right) \times \Omega_{1}} A^{i}\left(t, x, \mathbf{u}_{n}^{1}\right) z^{i} \mathrm{~d} x \mathrm{~d} t+\int_{\Omega_{1}} u_{0}^{i, 1} z^{i}(0) \mathrm{d} x\right\}+\int_{\left(0, T_{\max }\right) \times \Omega_{1}} \rho_{n}^{1} z^{2} \mathrm{~d} x \mathrm{~d} t, \\
\forall \mathbf{z} \in W^{1, \infty, \infty}\left(0, T_{\max } ; \mathbf{V}_{1}, \mathbf{V}_{1}^{\prime}\right) \quad \text { such that } \mathbf{z}\left(T_{\max }\right)=0, \quad(3.5) \\
\sum_{i=1}^{4}\left\{\int_{\left(0, T_{\max }\right) \times \Omega_{2}} \mu_{i} \nabla u_{n}^{i, 2} \cdot \nabla z^{i} \mathrm{~d} x \mathrm{~d} t+\int_{\left(0, T_{\max }\right) \times \Omega_{2}} \mathbf{w}_{i}^{2} \cdot \nabla u_{n}^{i, 2} z^{i} \mathrm{~d} x \mathrm{~d} t-\int_{0}^{T_{\max }}\left\langle\frac{\mathrm{d} z^{i}}{\mathrm{~d} t}(t), u_{n}^{i, 2}(t)\right\rangle_{\mathbf{V}_{2}^{\prime}, \mathbf{V}_{2}} \mathrm{~d} t\right\} \\
=\sum_{i=1}^{4} \int_{\left(0, T_{\max }\right) \times \Omega_{2}} A^{i}\left(t, x, \mathbf{u}_{n}^{2}\right) z^{i} \mathrm{~d} x \mathrm{~d} t+\frac{1}{\operatorname{meas}\left(\Omega_{1}\right)} \sum_{i=1}^{3} \int_{\Omega_{2}}\left[\int_{\Omega_{1}} u_{n}^{i, 1}\left(T_{n}^{1}\right) \mathrm{d} x\right] z^{i}(0) \mathrm{d} x+\int_{\left(0, T_{\max }\right) \times \Omega_{2}} \rho_{n}^{2} z^{2} \mathrm{~d} x \mathrm{~d} t, \\
\forall \mathbf{z} \in W^{1, \infty, \infty}\left(0, T_{\max } ; \mathbf{V}_{2}, \mathbf{V}_{2}^{\prime}\right) \quad \text { such that } \mathbf{z}\left(T_{\max }\right)=0 . \quad(3.6
\end{gathered}
$$

Passing to the limit in all the terms in (3.5) and in almost all the terms in (3.6) is a direct consequence of the convergences in (3.4). The only terms in (3.6) where we can not pass to the limit in an immediate way are those of the form $\int_{\Omega_{1}} u_{n}^{i, 1}\left(T_{n}^{1}\right) \mathrm{d} x$. However, for those terms the pass to the limit can be obtained by means of the change of variable $\tau=\frac{t}{T_{n}^{1}}$ in the state system corresponding to tank 1 . So, if we denote $v_{n}^{i, 1}(\tau)=u_{n}^{i, 1}\left(T_{n}^{1} \tau\right)=u_{n}^{i, 1}(t)$, this state system turns into:

$$
\begin{cases}\frac{\partial v_{n}^{i, 1}}{\partial \tau}+T_{n}^{1} \mathbf{w}_{i}^{1} \cdot \nabla v_{n}^{i, 1}-T_{n}^{1} \nabla \cdot\left(\mu_{i} \nabla v_{n}^{i, 1}\right)=T_{n}^{1} A^{i}\left(t, x, \mathbf{v}_{n}^{1}\right)+\delta_{2 i} T_{n}^{1} \rho_{n}^{1} & \text { in }(0,1) \times \Omega_{1}, \\ \frac{\partial v_{n}^{i, 1}}{\partial n}=0 & \text { on }(0,1) \times \partial \Omega_{1}, \\ v_{n}^{i, 1}(0)=u_{0}^{i, 1} & \text { in } \Omega_{1},\end{cases}
$$

where $\delta_{2 i}$ denotes the Kronecker's delta, that is, $\delta_{2 i}=1$ if $i=2$, and $\delta_{2 i}=0$ if $i \neq 2$.

Arguing as for equation (3.5) we can pass to the limit in above system, and obtain the existence of a subsequence of $\left\{\mathbf{v}_{n}^{1}\right\}_{n \in \mathbb{N}}$ (still denoted in the same way) converging to $\widetilde{\mathbf{v}}^{1}$, with $\widetilde{v}^{i, 1}(\tau)=\widetilde{u}^{i, 1}\left(\widetilde{T}^{1} \tau\right)$, in similar spaces as $\left\{\mathbf{u}_{n}^{1}\right\}_{n \in \mathbb{N}}$ in (3.4). In particular, we have that:

$$
\mathbf{v}_{n}^{1}(\tau) \rightarrow \widetilde{\mathbf{v}}^{1}(\tau) \text { in } \mathbf{H}_{1}, \quad \forall \tau \in[0,1] .
$$

But, this convergence implies, in a direct manner, that:

$$
\lim _{n \rightarrow \infty} \int_{\Omega_{1}} v_{n}^{i, 1}(1) \mathrm{d} x=\int_{\Omega_{1}} \widetilde{v}^{i, 1}(1) \mathrm{d} x
$$

or, equivalently, that:

$$
\lim _{n \rightarrow \infty} \int_{\Omega_{1}} u_{n}^{i, 1}\left(T_{n}^{1}\right) \mathrm{d} x=\int_{\Omega_{1}} \widetilde{u}^{i, 1}\left(\widetilde{T}^{1}\right) \mathrm{d} x .
$$

So, we have just proved that $\left(\widetilde{\mathbf{u}}^{1}, \widetilde{\mathbf{u}}^{2}\right)$ is the unique state associated to the control $\left(\widetilde{T}^{1}, \widetilde{T}^{2}, \widetilde{\rho}^{1}, \widetilde{\rho}^{2}\right)$.

On the other hand, from the properties of $\mathcal{V}_{a d}$, it is clear that $\left(\widetilde{T}^{1}, \widetilde{T}^{2}, \widetilde{\rho}^{1}, \widetilde{\rho}^{2}\right) \in \mathcal{V}_{a d}$. Thus, we are only left to prove that $\left(\widetilde{\mathbf{u}}^{1}, \widetilde{\mathbf{u}}^{2}\right)$ satisfy the constraints (2.8). The first and third constraints are satisfied, as a direct consequence of convergence (3.9). For demonstrating the verification of the other two constraints we only need 
to use the new change of variable $\tau=\frac{t}{T_{n}^{2}}$ in the state system corresponding to Tank 2. So, if we denote $v_{n}^{i, 2}(\tau)=u_{n}^{i, 2}\left(T_{n}^{2} \tau\right)=u_{n}^{i, t}(t)$, by similar arguments to those given for the state system corresponding to Tank 1, we obtain that:

$$
\lim _{n \rightarrow \infty} \int_{\Omega_{2}} u_{n}^{i, 2}\left(T_{n}^{2}\right) \mathrm{d} x=\int_{\Omega_{2}} \widetilde{u}^{i, 2}\left(\widetilde{T}^{2}\right) \mathrm{d} x
$$

from which we conclude that constraints (2.8) are satisfied.

Moreover, by convergences (3.4) and (3.10), we deduce that:

$$
\lim _{n \rightarrow \infty} J\left(T_{n}^{1}, T_{n}^{2}, \rho_{n}^{1}, \rho_{n}^{2}\right)=J\left(\widetilde{T}^{1}, \widetilde{T}^{2}, \widetilde{\rho}^{1}, \widetilde{\rho}^{2}\right),
$$

i.e. $\left(\widetilde{T}^{1}, \widetilde{T}^{2}, \widetilde{\rho}^{1}, \widetilde{\rho}^{2}\right)$ is a solution of the time optimal control problem $(\mathcal{P})$.

\section{Optimality COnditions}

In this section we will obtain the necessary first order optimality conditions for characterizing the solutions of the time optimal control problem $(\mathcal{P})$. As noted in the previous section, since two of the control variables are the permanence times $\left(T^{1}, T^{2}\right)$, we will need to guarantee the differentiability of the states with respect to the time $t$. So, we will need to impose the more restrictive hypotheses (3.2) on the fluid velocities and temperatures to assure the existence of optimality conditions. To obtain these optimality conditions we will use a change of time variable (introducing a new control $v$ in the spirit of the original ideas of Raymond and Zidani [15]), since a simple scaling of time has shown to be insufficient for our purposes. It is worthwhile remarking here that the time transformation by introducing a new control $v$ had been previously introduced - within the ordinary differential equations framework - in the classical book of Zeidler [21], for instance.

For a better organization and understanding of the results exposed in this section, we will divide it into two subsections. In the first one we will analyze how to obtain the optimality conditions for a new optimal control problem $(\widetilde{\mathcal{P}})$ with fixed final time, obtained from the problem $(\mathcal{P})$ via a change of the time variable. In the second subsection we will use the results obtained in the first one in order to derive the optimality conditions for the original time optimal control problem $(\mathcal{P})$.

\subsection{The problem with fixed final time}

For any given time interval $[0, T] \subset \mathbb{R}$ with $T>0$, and for $\mathbb{R}_{*}^{+}=(0, \infty)$, we will consider a function $v \in \mathcal{C}\left([0,1] ; \mathbb{R}_{*}^{+}\right)$such that:

$$
\int_{0}^{1} v(\zeta) \mathrm{d} \zeta=T
$$

Associated to above function, we will define the following change of variable:

$$
t=\phi(\tau):=\int_{0}^{\tau} v(\zeta) \mathrm{d} \zeta, \quad \tau \in[0,1]
$$

that implies $\frac{\mathrm{d} \phi}{\mathrm{d} \tau}(\tau)=v(\tau)$, which will give rise to the new state equations.

Then if for each one of both tanks $\Omega_{j}, j=1,2$, we consider the corresponding change of variable $t=\phi^{j}(\tau)=$ $\int_{0}^{\tau} v^{j}(\xi) \mathrm{d} \xi, \tau \in[0,1]$, we will introduce the following notations:

$$
\mathbf{y}^{j}(\tau):=\left(\mathbf{u}^{j} \circ \phi^{j}\right)(\tau)=\mathbf{u}^{j}(t), \quad \varrho^{j}(\tau):=\left(\rho^{j} \circ \phi^{j}\right)(\tau)=\rho^{j}(t), \quad j=1,2 .
$$

Applying above changes of variable to our original optimal control problem with varying final time $(\mathcal{P})$, we can rewrite it under the following alternative form of an optimal control problem with fixed final time: 
- Controls: The four new design variables will be the functions $v^{j}(\tau), j=1,2$, for the changes of time variables, and the corresponding transformed quantities $\varrho^{j}(\tau, x)$ of phytoplankton added in the tank $\Omega_{j}$, $j=1,2$, along the new time interval $(0,1)$.

- State systems: We consider the two transformed state systems for the concentrations of nitrogen, phytoplankton, zooplankton, and organic detritus in each one of the tanks, and two new state equations for the corresponding changes of time variable in each one of the tanks.

- Species in tank $\Omega_{1}$ :

$$
\begin{cases}\frac{\partial y^{i, 1}}{\partial \tau}+v^{1}(\tau) \mathbf{w}_{i}^{1}\left(\phi^{1}(\tau), x\right) \cdot \nabla y^{i, 1}-v^{1}(\tau) \nabla \cdot\left(\mu_{i} \nabla y^{i, 1}\right) & \\ \quad=v^{1}(\tau) A^{i}\left(\phi^{1}(\tau), x, \mathbf{y}^{1}\right)+\delta_{2 i} v^{1}(\tau) \varrho^{1}(\tau) & \text { in }(0,1) \times \Omega_{1}, \\ \frac{\partial y^{i, 1}}{\partial n}=0 & \text { on }(0,1) \times \partial \Omega_{1}, \\ y^{i, 1}(0)=u_{0}^{i, 1} & \text { in } \Omega_{1} .\end{cases}
$$

- Species in tank $\Omega_{2}$ :

$$
\begin{cases}\frac{\partial y^{i, 2}}{\partial \tau}+v^{2}(\tau) \mathbf{w}_{i}^{2}\left(\phi^{2}(\tau), x\right) \cdot \nabla y^{i, 1}-v^{2}(\tau) \nabla \cdot\left(\mu_{i} \nabla y^{i, 2}\right) & \\ \quad=v^{2}(\tau) A^{i}\left(\phi^{2}(\tau), x, \mathbf{y}^{2}\right)+\delta_{2 i} v^{2}(\tau) \varrho^{2}(\tau) & \text { in }(0,1) \times \Omega_{2}, \\ \frac{\partial y^{i, 2}}{\partial n}=0 & \text { on }(0,1) \times \partial \Omega_{2}, \\ y^{i, 2}(0)=\frac{1}{\operatorname{meas}\left(\Omega_{1}\right)} \mathbf{M}_{1}^{i}\left(\mathbf{y}^{1}(1)\right) & \text { in } \Omega_{2} .\end{cases}
$$

- Change of time variable in tank $\Omega_{1}$ :

$$
\left\{\begin{array}{l}
\frac{\mathrm{d} \phi^{1}}{\mathrm{~d} \tau}(\tau)=v^{1}(\tau) \quad \text { in }(0,1), \\
\phi^{1}(0)=0
\end{array}\right.
$$

- Change of time variable in tank $\Omega_{2}$ :

$$
\left\{\begin{array}{l}
\frac{\mathrm{d} \phi^{2}}{\mathrm{~d} \tau}(\tau)=v^{2}(\tau) \quad \text { in }(0,1) \\
\phi^{2}(0)=0
\end{array}\right.
$$

- Objective function:

$$
\widetilde{J}\left(v^{1}, v^{2}, \varrho^{1}, \varrho^{2}\right)=N_{1} \int_{0}^{1}\left(v^{1}(\tau)+v^{2}(\tau)\right) \mathrm{d} \tau+\frac{N_{2}}{\operatorname{meas}\left(\Omega_{2}\right)} \int_{\Omega_{2}} y^{2,2}(1) \mathrm{d} x .
$$

\section{- State constraints:}

$$
\left\{\begin{array}{l}
\widetilde{B}^{1}\left(v^{1}, v^{2}, \varrho^{1}, \varrho^{2}\right)=\frac{1}{\operatorname{meas}\left(\Omega_{1}\right)} \int_{\Omega_{1}} y^{1,1}(1) \mathrm{d} x \leq \sigma_{1}, \\
\widetilde{B}^{2}\left(v^{1}, v^{2}, \varrho^{1}, \varrho^{2}\right)=\frac{1}{\operatorname{meas}\left(\Omega_{2}\right)} \int_{\Omega_{2}} y^{1,2}(1) \mathrm{d} x \leq \sigma_{2} \\
\widetilde{B}^{3}\left(v^{1}, v^{2}, \varrho^{1}, \varrho^{2}\right)=\frac{1}{\operatorname{meas}\left(\Omega_{1}\right)} \int_{\Omega_{1}} y^{4,1}(1) \mathrm{d} x \geq \theta_{1} \\
\widetilde{B}^{4}\left(v^{1}, v^{2}, \varrho^{1}, \varrho^{2}\right)=\frac{1}{\operatorname{meas}\left(\Omega_{2}\right)} \int_{\Omega_{2}} y^{4,2}(1) \mathrm{d} x \geq \theta_{2} .
\end{array}\right.
$$

- Control constraints: As commented in above paragraphs, functions $\left(v^{1}, v^{2}\right)$ corresponding to the changes of time variable must lie in the set

$$
\mathcal{W}_{a d}^{1}=\left\{\left(v^{1}, v^{2}\right) \in \mathcal{C}\left([0,1] ; \mathbb{R}^{+}\right)^{2}: T_{\min } \leq v^{1}(\tau), v^{2}(\tau) \leq T_{\max }, \forall \tau \in[0,1]\right\} .
$$


Moreover, the transformed quantities $\left(\varrho^{1}, \varrho^{2}\right)$ of phytoplankton added to both tanks must lie in the set

$$
\begin{aligned}
\mathcal{W}_{a d}^{2}= & \left\{\left(\varrho^{1}, \varrho^{2}\right) \in L^{2}\left((0,1) \times \Omega_{1}\right) \times L^{2}\left((0,1) \times \Omega_{2}\right):\right. \\
& 0 \leq \varrho^{1}(\tau, x) \leq K \text { a.e. } \quad(\tau, x) \in(0,1) \times \Omega_{1} \\
& \left.0 \leq \varrho^{2}(\tau, x) \leq K \text { a.e. } \quad(\tau, x) \in(0,1) \times \Omega_{2}\right\}
\end{aligned}
$$

So, if we denote $\mathcal{W}_{a d}=\mathcal{W}_{a d}^{1} \times \mathcal{W}_{a d}^{2}$, which is a closed, bounded, convex, and nonempty subset of $\mathcal{C}\left([0,1] ; \mathbb{R}^{+}\right)^{2} \times L^{2}\left((0,1) \times \Omega_{1}\right) \times L^{2}\left((0,1) \times \Omega_{2}\right)$, the control must satisfy $\left(v^{1}, v^{2}, \varrho^{1}, \varrho^{2}\right) \in \mathcal{W}_{a d}$.

Thus, the formulation of the optimal control problem with fixed final time, denoted by $(\widetilde{\mathcal{P}})$, will be the following:

$(\widetilde{\mathcal{P}}) \quad \inf \left\{\widetilde{J}\left(v^{1}, v^{2}, \varrho^{1}, \varrho^{2}\right)\right.$ such that $\left(v^{1}, v^{2}, \varrho^{1}, \varrho^{2}\right) \in \mathcal{W}_{a d}$ and $\left(\mathbf{y}^{1}, \mathbf{y}^{2}, \phi^{1}, \phi^{2}\right)$

satisfies (4.4)-(4.5), (4.6)-(4.7) and (4.9)\}.

By introducing the above changes of time variable we actually transform time into two new state variables $\phi^{j}$, $j=1,2$, which will be determined by the new control variables $v^{j}, j=1,2$, by means of the initial value problems (4.6)-(4.7).

The existence and uniqueness of solution of the new state systems (4.4)-(4.5) and (4.6)-(4.7) is again a direct application of well-known techniques:

Lemma 4.1. For any given element $\left(v^{1}, v^{2}, \varrho^{1}, \varrho^{2}\right) \in \mathcal{W}_{\text {ad }}$, there exists $\left(\mathbf{y}^{1}, \mathbf{y}^{2}, \phi^{1}, \phi^{2}\right) \in\left(W^{1,2,2}\left(0,1 ; \mathbf{V}_{1}, \mathbf{V}_{1}^{\prime}\right) \cap\right.$ $\left.\left[L^{\infty}\left((0,1) \times \Omega_{1}\right)\right]^{4}\right) \times\left(W^{1,2,2}\left(0,1 ; \mathbf{V}_{2}, \mathbf{V}_{2}^{\prime}\right) \cap\left[L^{\infty}\left((0,1) \times \Omega_{2}\right)\right]^{4}\right) \times \mathcal{C}^{1}([0,1] ; \mathbb{R})^{2}$ the unique solution of the state systems (4.4)-(4.5) and (4.6)-(4.7).

Moreover, for $\left(\mathbf{y}^{1}, \mathbf{y}^{2}\right)$, we have the estimates:

$$
\begin{aligned}
& \left\|\mathbf{y}^{j}\right\|_{W^{1,2,2}\left(0,1 ; \mathbf{V}_{j}, \mathbf{V}_{j}^{\prime}\right) \cap\left[L^{\infty}\left((0,1) \times \Omega_{j}\right)\right]^{4}} \leq C, \quad j=1,2, \\
& 0 \leq \mathbf{y}^{j}(\tau, x) \leq C, \quad \text { a.e. } \quad(\tau, x) \in(0,1) \times \Omega_{j}, \quad j=1,2,
\end{aligned}
$$

where $C=C\left(T_{\min }, T_{\max }, M\right)$ is a non-negative constant only depending on $T_{\min }, T_{\max }$ and $M$.

Proof. Existence, uniqueness and boundedness of solution $\left(\mathbf{y}^{1}, \mathbf{y}^{2}\right)$ for the state systems (4.4)-(4.5) can be obtained by the same arguments to those used in the proof of existence, uniqueness and boundedness of solution for the original state systems (2.4)-(2.5). The only difference here is that the diffusive part of the partial differential operator (that is, the terms of the form $\left.v^{j}(\tau) \nabla \cdot\left(\mu_{i} \nabla y^{i, j}\right)\right)$ are affected by time-varying coefficients: However, this fact does not represent any difficulty in our arguing, since those coefficients $v^{j} \in L^{\infty}(0,1)$ are bounded from below by a strictly positive value $T_{\min }$.

Finally, existence and uniqueness of solution $\left(\phi^{1}, \phi^{2}\right)$ of the state equations (4.6)-(4.7) can be obtained from classical results for ordinary differential equations.

Next, following the ideas of Raymond and Zidani [15], we will prove a result stating the equivalence between the new optimal control problem $(\widetilde{\mathcal{P}})$ and original time optimal control problem $(\mathcal{P})$. In order to this, we need to introduce the following definition:

Definition 4.2. We will say that $\left(v^{1}, v^{2}, \varrho^{1}, \varrho^{2}\right) \in \mathcal{W}_{a d}$ is a feasible control for the optimal control problem $(\widetilde{\mathcal{P}})$ if the associated state $\left(\mathbf{y}^{1}, \mathbf{y}^{2}, \phi^{1}, \phi^{2}\right)$, solution of systems (4.4)-(4.5) and (4.6)-(4.7), satisfies the constraints (4.9). 
Theorem 4.3. Problems $(\widetilde{\mathcal{P}})$ and $(\mathcal{P})$ are equivalent in the following sense:

(a) Let $\left(v^{1}, v^{2}, \varrho^{1}, \varrho^{2}\right) \in \mathcal{W}_{a d}$ be a feasible control for problem $(\widetilde{\mathcal{P}})$. Then, there exists a feasible control $\left(T^{1}, T^{2}, \rho^{1}, \rho^{2}\right) \in \mathcal{V}_{\text {ad }}$ for problem $(\mathcal{P})$, such that:

$$
\begin{aligned}
& \varrho^{j}=\rho^{j} \circ \phi^{j}, \quad \phi^{j}(1)=T^{j}, \quad j=1,2, \\
& \widetilde{J}\left(v^{1}, v^{2}, \varrho^{1}, \varrho^{2}\right)=J\left(T^{1}, T^{2}, \rho^{1}, \rho^{2}\right) .
\end{aligned}
$$

(b) Let $\left(T^{1}, T^{2}, \rho^{1}, \rho^{2}\right) \in \mathcal{V}_{a d}$ be a feasible control for problem $(\mathcal{P})$. Then, for all $\left(v^{1}, v^{2}\right) \in \mathcal{C}\left([0,1] ; \mathbb{R}^{+}\right)^{2}$, such that $T_{\min } \leq v^{1}(\tau), v^{2}(\tau) \leq T_{\max }, \forall \tau \in[0,1]$, and $T^{j}=\int_{0}^{1} v^{j}(\xi) \mathrm{d} \xi$, the element $\left(v^{1}, v^{2}, \varrho^{1}, \varrho^{2}\right)$ with $\varrho^{j}=\rho^{j} \circ \phi^{j}$ is a feasible control for problem $(\widetilde{\mathcal{P}})$. Moreover,

$$
J\left(T^{1}, T^{2}, \rho^{1}, \rho^{2}\right)=\widetilde{J}\left(v^{1}, v^{2}, \varrho^{1}, \varrho^{2}\right) .
$$

Proof. Since part (b) of the theorem is a direct consequence of above computations in this subsection, we are only left to prove part (a). Let $\left(v^{1}, v^{2}, \varrho^{1}, \varrho^{2}\right) \in \mathcal{W}_{a d}$ be a feasible control for problem $(\widetilde{\mathcal{P}})$. Since $v^{j}(\tau) \geq T_{\text {min }}>0$, $\forall \tau \in[0,1], j=1,2$, we know that the mappings $\phi^{j}(\tau)=\int_{0}^{\tau} v^{j}(\xi) \mathrm{d} \xi, j=1,2$, are strictly increasing in the interval $[0,1]$. Thus, for $j=1,2$, the mapping $\phi^{j}:[0,1] \rightarrow\left[0, \phi^{j}(1)\right]$ is bijective, which allows us to define the inverse mapping $\left(\phi^{j}\right)^{-1}:\left[0, \phi^{j}(1)\right] \rightarrow[0,1]$. So, let us consider the element $\left(T^{1}, T^{2}, \rho^{1}, \rho^{2}\right)$ defined by:

$$
\begin{aligned}
& T^{j}=\phi^{j}(1), \quad j=1,2, \\
& \rho^{j}(t, x)=\left\{\begin{array}{ll}
\varrho\left(\left(\phi^{j}\right)^{-1}(t), x\right), & \text { if } t \leq \phi^{j}(1) \\
0, & \text { if } t>\phi^{j}(1)
\end{array} \quad j=1,2 .\right.
\end{aligned}
$$

Now it is a direct computation to demonstrate that the element $\left(T^{1}, T^{2}, \rho^{1}, \rho^{2}\right)$ above defined is a feasible control for problem $(\mathcal{P})$.

The key point in the proof of the equivalence between both control problems relies in the choice of the controls $\left(v^{1}, v^{2}\right)$ such that they are strictly positive. Our arguments would not remain true if we substitute the condition $T_{\min } \leq v^{1}(\tau), v^{2}(\tau) \leq T_{\max }, \forall \tau \in[0,1]$, in the definition of $\mathcal{W}_{a d}$ by the (more natural at a first sight) condition $T_{\min } \leq \int_{0}^{1} v^{1}(\tau) \mathrm{d} \tau, \int_{0}^{1} v^{2}(\tau) \mathrm{d} \tau \leq T_{\max }$, due to the fact that, under this latter condition, it is possible to find controls $v^{j} \in \mathcal{C}\left([0,1] ; \mathbb{R}^{+}\right)$but taking null values in a subinterval of $[0,1]$. In this case, we could not assure the mapping $\phi^{j}$ to be bijective, and both control problems should not be necessarily equivalent.

The most evident advantage of dealing with the optimal control problem $(\widetilde{\mathcal{P}})$ instead of the original time optimal control problem $(\mathcal{P})$ is that $(\widetilde{\mathcal{P}})$ is a problem with fixed final time, which will allow us to obtain, in a simpler way, the optimality conditions for our problem.

Let us derive now the optimality conditions for the optimal control $(\widetilde{\mathcal{P}})$. In order to do this, we begin by an analysis of the behavior of the derivatives of the nonlinear operator $\mathbf{A}=\left(A^{1}, A^{2}, A^{3}, A^{4}\right): \mathbb{R}^{+} \times \Omega \times\left[\mathbb{R}^{+}\right]^{4} \longrightarrow \mathbb{R}^{4}$ with respect to the time $t \in \mathbb{R}^{+}$and to the real variable $\mathbf{u} \in\left[\mathbb{R}^{+}\right]^{4}$. On one hand, it is clear that, $\forall \mathbf{u} \in\left[\mathbb{R}^{+}\right]^{4}$, the Jacobian $D_{\mathbf{u}} \mathbf{A}(t, x, \mathbf{u})$ will be given by the following matrix with Caratheodory coefficients in $\mathbb{R}^{+} \times \Omega \times\left[\mathbb{R}^{+}\right]^{4}$ :

$$
\left(\begin{array}{cccc}
-C_{n c} L(t, x) \frac{K_{N}}{\left(K_{N}+u^{1}\right)^{2}} u^{2} & -C_{n c} L(t, x) \frac{u^{1}}{K_{N}+u^{1}}+C_{n c} K_{r} & 0 & C_{n c} K_{r d} \Theta^{\theta(t, x)-20} \\
L(t, x) \frac{K_{N}}{\left(K_{N}+u^{1}\right)^{2}} u^{2} & L(t, x) \frac{u^{1}}{K_{N}+u^{1}}-\left(K_{r}+K_{m f}\right)-K_{z} \frac{K_{F}}{\left(K_{F}+u^{2}\right)^{2}} u^{3} & -K_{z} \frac{u^{2}}{K_{F}+u^{2}} & 0 \\
0 & C_{f z} K_{z} \frac{K_{F}}{\left(K_{F}+u^{2}\right)^{2}} u^{3} & C_{f z} K_{z} \frac{u^{2}}{K_{F}+u^{2}}-K_{m z} & 0 \\
0 & K_{m f} & K_{m z} & -K_{r d} \Theta^{\theta(t, x)-20}
\end{array}\right)
$$


and that, $\forall t \in \mathbb{R}^{+}$, the derivative $D_{t} \mathbf{A}(t, x, \mathbf{u})$ will be given by the following vector with Caratheodory components in $\mathbb{R}^{+} \times \Omega \times\left[\mathbb{R}^{+}\right]^{4}$ :

$$
D_{t} \mathbf{A}(t, x, \mathbf{u})=\left(\begin{array}{c}
-C_{n c} \frac{\partial L}{\partial t}(t, x) \frac{u^{1}}{K_{N}+u^{1}} u^{2}+C_{n c} K_{r d} \Theta^{\theta(t, x)-20} \ln (\Theta) \frac{\partial \theta}{\partial t}(t, x) u^{4} \\
\frac{\partial L}{\partial t}(t, x) \frac{u^{1}}{K_{N}+u^{1}} u^{2} \\
0 \\
-K_{r d} \Theta^{\theta(t, x)-20} \ln (\Theta) \frac{\partial \theta}{\partial t}(t, x) u^{4}
\end{array}\right)
$$

So, for $\theta$ satisfying hypothesis $(3.2)$, we have for $i, j=1, \ldots, 4$ :

$$
\begin{aligned}
& \left|\frac{\partial A^{i}}{\partial u^{j}}(t, x, \mathbf{u})\right| \leq c_{0}+\sum_{k=1}^{4} c_{k} u^{k}, \quad \forall(t, x, \mathbf{u}) \in \mathbb{R}^{+} \times \Omega \times\left[\mathbb{R}^{+}\right]^{4}, \\
& \left|\frac{\partial A^{i}}{\partial t}(t, x, \mathbf{u})\right| \leq \sum_{k=1}^{4} c_{k} u^{k}, \quad \forall(t, x, \mathbf{u}) \in \mathbb{R}^{+} \times \Omega \times\left[\mathbb{R}^{+}\right]^{4},
\end{aligned}
$$

with $c_{0}, c_{1}, \ldots, c_{4}$ non-negative constants.

Then, we can demonstrate the following result stating the differentiability of the state system with respect to the time control variable $v$ :

Theorem 4.4. We consider, for any given $0<C_{1}<C_{2}<\infty$, the following closed, convex subset of $\mathcal{C}\left([0,1] ; \mathbb{R}_{*}^{+}\right)$:

$$
\Psi_{C_{1}, C_{2}}=\left\{v \in \mathcal{C}\left([0,1] ; \mathbb{R}^{+}\right): C_{1} \leq v(\tau) \leq C_{2}, \quad \forall \tau \in[0,1]\right\}
$$

and define, $\forall v \in \Psi_{C_{1}, C_{2}}$, the element $\mathbf{u}_{v} \in W^{1,2,2}\left(0,1 ; \mathbf{V}, \mathbf{V}^{\prime}\right) \cap\left[L^{\infty}((0,1) \times \Omega)\right]^{4}$ as the solution of the system, for $i=1, \ldots, 4$ :

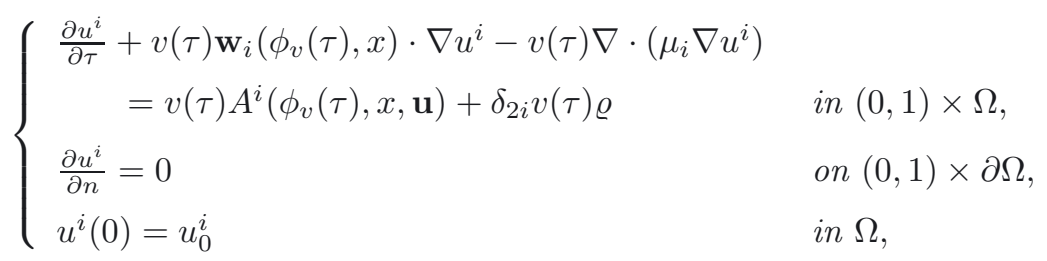

where initial condition $\mathbf{u}_{0} \in\left[L^{\infty}(\Omega)\right]^{4}$ is such that $0 \leq u_{0}^{i}(x) \leq M$ a.e. $x \in \Omega, i=1, \ldots, 4$, distributed control $\varrho \in L^{2}((0,1) \times \Omega)$ is such that $0 \leq \varrho(\tau, x) \leq M$ a.e. $(\tau, x) \in(0,1) \times \Omega$, water velocity $\mathbf{w}$ and temperature $\theta$ satisfy hypothesis $(3.2)$, and $\phi_{v} \in \overline{\mathcal{C}}^{1}([0,1] ; \mathbb{R})$ is the solution of the following initial value problem:

$$
\left\{\begin{array}{l}
\frac{\mathrm{d} \phi_{v}}{\mathrm{~d} \tau}(\tau)=v(\tau) \quad \text { in }(0,1) \\
\phi_{v}(0)=0
\end{array}\right.
$$

If $\forall v_{1}, v_{2} \in \Psi_{C_{1}, C_{2}}$ and $\forall \delta \in(0,1)$ we denote $v_{\delta}=v_{1}+\delta\left(v_{2}-v_{1}\right)$, then we have that $\mathbf{u}_{v_{\delta}}$ verifies the equality:

$$
\mathbf{u}_{v_{\delta}}=\mathbf{u}_{v_{1}}+\delta \mathbf{h}+\mathbf{r}_{\delta}
$$


where $\mathbf{h} \in W^{1,2,2}\left(0,1 ; \mathbf{V}, \mathbf{V}^{\prime}\right)$ is the solution of the system, for $i=1, \ldots, 4$ :

$$
\begin{cases}\frac{\partial h^{i}}{\partial \tau}+v_{1}(\tau) \mathbf{w}_{i}\left(\phi_{v_{1}}(\tau), x\right) \cdot \nabla h^{i}-v_{1}(\tau) \nabla \cdot\left(\mu_{i} \nabla h^{i}\right)=v_{1}(\tau)\left[D_{\mathbf{u}} \mathbf{A}\left(\phi_{v_{1}}(\tau), x, \mathbf{u}_{v_{1}}\right) \mathbf{h}\right]_{i} & \\ \quad+\left(\phi_{v_{2}}(\tau)-\phi_{v_{1}}(\tau)\right)\left\{v_{1}(\tau)\left[D_{t} \mathbf{A}\left(\phi_{v_{1}}(\tau), x, \mathbf{u}_{v_{1}}\right)\right]_{i}-v_{1}(\tau) D_{t} \mathbf{w}\left(\phi_{v_{1}}(\tau), x\right) \cdot \nabla u_{v_{1}}^{i}\right\} & \\ \quad+\left(v_{2}(\tau)-v_{1}(\tau)\right)\left\{\nabla \cdot\left(\mu_{i} \nabla u_{v_{1}}^{i}\right)-\mathbf{w}_{i}\left(\phi_{v_{1}}(\tau), x\right) \cdot \nabla u_{v_{1}}^{i}+A^{i}\left(\phi_{v_{1}}(\tau), x, \mathbf{u}_{v_{1}}\right)+\delta_{2 i} \varrho\right\} & \text { in }(0,1) \times \Omega, \\ \frac{\partial h^{i}}{\partial n}=0 & \text { on }(0,1) \times \partial \Omega, \\ h^{i}(0)=0 & \text { in } \Omega,\end{cases}
$$

and where $\mathbf{r}_{\delta} \in W^{1,2,2}\left(0,1 ; \mathbf{V}, \mathbf{V}^{\prime}\right)$ is such that:

$$
\lim _{\delta \rightarrow 0} \frac{1}{\delta}\left\|\mathbf{r}_{\delta}\right\|_{W^{1,2,2}\left(0,1 ; \mathbf{V}, \mathbf{V}^{\prime}\right)}=0
$$

Proof. For system (4.17) we can obtain, arguing as in the first part of Lemma 4.1, the existence of a unique solution $\mathbf{h} \in W^{1,2,2}\left(0,1 ; \mathbf{V}, \mathbf{V}^{\prime}\right) \cap\left[L^{\infty}((0,1) \times \Omega)\right]^{4}$. Then, if we define $\mathbf{z}_{\delta}=\frac{\mathbf{u}_{v_{\delta}}-\mathbf{u}_{v_{1}}}{\delta}-\mathbf{h}=\frac{\mathbf{r}_{\delta}}{\delta}$, we have that $\mathbf{z}_{\delta} \in W^{1,2,2}\left(0,1 ; \mathbf{V}, \mathbf{V}^{\prime}\right)$ satisfies the system, for $i=1, \ldots, 4$ :

$$
\left\{\begin{array}{l}
\frac{\partial z_{\delta}^{i}}{\partial \tau}+\frac{v_{\delta}(\tau)}{\delta} \mathbf{w}_{i}\left(\phi_{v_{\delta}}(\tau), x\right) \cdot \nabla u_{v_{\delta}}^{i}-\frac{v_{1}(\tau)}{\delta} \mathbf{w}_{i}\left(\phi_{v_{1}}(\tau), x\right) \cdot \nabla u_{v_{1}}^{i}-v_{1}(\tau) \mathbf{w}_{i}\left(\phi_{v_{1}}(\tau), x\right) \cdot \nabla h^{i} \\
-\frac{v_{\delta}(\tau)}{\delta} \nabla \cdot\left(\mu_{i} \nabla u_{v_{\delta}}^{i}\right)+\frac{v_{1}(\tau)}{\delta} \nabla \cdot\left(\mu_{i} \nabla u_{v_{1}}^{i}\right)+v_{1}(\tau) \nabla \cdot\left(\mu_{i} \nabla h^{i}\right) \\
=\frac{v_{\delta}(\tau)}{\delta}\left(A^{i}\left(\phi_{v_{\delta}}(\tau), x, \mathbf{u}_{v_{\delta}}\right)\right)-\frac{v_{1}(\tau)}{\delta}\left(A^{i}\left(\phi_{v_{1}}(\tau), x, \mathbf{u}_{v_{1}}\right)\right)-v_{1}(\tau)\left[D_{\mathbf{u}} \mathbf{A}\left(\phi_{v_{1}}(\tau), x, \mathbf{u}_{v_{1}}\right) \mathbf{h}\right]_{i} \\
-\left(v_{2}(\tau)-v_{1}(\tau)\right)\left\{\nabla \cdot\left(\mu_{i} \nabla u_{v_{1}}^{i}\right)-\mathbf{w}_{i}\left(\phi_{v_{1}}(\tau), x\right) \cdot \nabla u_{v_{1}}^{i}+A^{i}\left(\phi_{v_{1}}(\tau), x, \mathbf{u}_{v_{1}}\right)\right\} \\
-\left(\phi_{v_{2}}(\tau)-\phi_{v_{1}}(\tau)\right)\left\{v_{1}(\tau)\left[D_{t} \mathbf{A}\left(\phi_{v_{1}}(\tau), x, \mathbf{u}_{v_{1}}\right)\right]_{i}-v_{1}(\tau) D_{t} \mathbf{w}\left(\phi_{v_{1}}(\tau), x\right) \cdot \nabla u_{v_{1}}^{i}\right\} \quad \text { in }(0,1) \times \Omega \\
\frac{\partial z_{\delta}^{i}}{\partial n}=0 \quad \text { on }(0,1) \times \partial \Omega \\
z_{\delta}^{i}(0)=0 \quad \text { in } \Omega .
\end{array}\right.
$$

To complete the proof we only have to demonstrate that $\lim _{\delta \rightarrow 0}\left\|\mathbf{z}_{\delta}\right\|_{W^{1,2,2}\left(0,1 ; \mathbf{V}, \mathbf{V}^{\prime}\right)}=0$. In order to obtain this, we consider the following Taylor series expansions valid a.e. $(\tau, x) \in(0,1) \times \Omega$, for $i=1, \ldots, 4$ :

$$
\begin{aligned}
& A^{i}\left(\phi_{v_{\delta}}(\tau), x, \mathbf{u}_{v_{\delta}}(\tau, x)\right)-A^{i}\left(\phi_{v_{1}}(\tau), x, \mathbf{u}_{v_{1}}(\tau, x)\right) \\
& =\int_{0}^{1} \frac{\partial A^{i}}{\partial t}\left(\phi_{v_{1}}(\tau)+\theta\left(\phi_{v_{\delta}}(\tau)-\phi_{v_{1}}(\tau)\right), x, \mathbf{u}_{v_{1}}(\tau, x)\right)\left(\phi_{v_{\delta}}(\tau)-\phi_{v_{1}}(\tau)\right) \mathrm{d} \theta \\
& \quad+\int_{0}^{1} \nabla_{\mathbf{u}} A^{i}\left(\phi_{v_{\delta}}(\tau), x, \mathbf{u}_{v_{1}}(\tau, x)+\theta\left(\mathbf{u}_{v_{\delta}}(\tau, x)-\mathbf{u}_{v_{1}}(\tau, x)\right)\right) \cdot\left(\mathbf{u}_{v_{\delta}}(\tau, x)-\mathbf{u}_{v_{1}}(\tau, x)\right) \mathrm{d} \theta \\
& \mathbf{w}_{i}\left(\phi_{v_{\delta}}(\tau), x\right)-\mathbf{w}_{i}\left(\phi_{v_{1}}(\tau), x\right)=\int_{0}^{1} D_{t} \mathbf{w}\left(\phi_{v_{1}}(\tau)+\theta\left(\phi_{v_{\delta}}(\tau)-\phi_{v_{1}}(\tau)\right), x\right)\left(\phi_{v_{\delta}}(\tau)-\phi_{v_{1}}(\tau)\right) \mathrm{d} \theta
\end{aligned}
$$


Thus, bearing in mind that $\frac{v_{\delta}}{\delta}-\frac{v_{1}}{\delta}=v_{2}-v_{1}$, from (4.20)-(4.21) we have that a.e. $(\tau, x) \in(0,1) \times \Omega$, for $i=1, \ldots, 4$ :

$$
\begin{aligned}
\frac{v_{\delta}(\tau)}{\delta} A^{i}\left(\phi_{v_{\delta}}(\tau), x, \mathbf{u}_{v_{\delta}}(\tau, x)\right)-\frac{v_{1}(\tau)}{\delta} A^{i}\left(\phi_{v_{1}}(\tau), x, \mathbf{u}_{v_{1}}(\tau, x)\right)=\left(v_{2}(\tau)-v_{1}(\tau)\right) A^{i}\left(\phi_{v_{1}}(\tau), x, \mathbf{u}_{v_{1}}(\tau, x)\right) \\
\quad+\frac{v_{\delta}(\tau)}{\delta} \int_{0}^{1} \frac{\partial A^{i}}{\partial t}\left(\phi_{v_{1}}(\tau)+\theta\left(\phi_{v_{\delta}}(\tau)-\phi_{v_{1}}(\tau)\right), x, \mathbf{u}_{v_{1}}(\tau, x)\right)\left(\phi_{v_{\delta}}(\tau)-\phi_{v_{1}}(\tau)\right) \mathrm{d} \theta \\
\quad+\frac{v_{\delta}(\tau)}{\delta} \int_{0}^{1} \nabla_{\mathbf{u}} A^{i}\left(\phi_{v_{\delta}}(\tau), x, \mathbf{u}_{v_{1}}(\tau, x)+\theta\left(\mathbf{u}_{v_{\delta}}(\tau, x)-\mathbf{u}_{v_{1}}(\tau, x)\right)\right) \cdot\left(\mathbf{u}_{v_{\delta}}(\tau, x)-\mathbf{u}_{v_{1}}(\tau, x)\right) \mathrm{d} \theta
\end{aligned}
$$

$$
\begin{aligned}
\frac{v_{\delta}(\tau)}{\delta} \mathbf{w}_{i}\left(\phi_{v_{\delta}}(\tau), x\right) \cdot \nabla & u_{v_{\delta}}^{i}(\tau, x)-\frac{v_{1}(\tau)}{\delta} \mathbf{w}_{i}\left(\phi_{v_{1}}(\tau), x\right) \cdot \nabla u_{v_{1}}^{i}(\tau, x)=\left(v_{2}(\tau)-v_{1}(\tau)\right) \mathbf{w}_{i}\left(\phi_{v_{1}}(\tau), x\right) \cdot \nabla u_{v_{1}}^{i}(\tau, x) \\
& +\frac{v_{\delta}(\tau)}{\delta} \mathbf{w}_{i}\left(\phi_{v_{\delta}}(\tau), x\right) \cdot \nabla\left(u_{v_{\delta}}^{i}-u_{v_{1}}^{i}\right)(\tau, x) \\
& +\frac{v_{\delta}(\tau)}{\delta} \int_{0}^{1} D_{t} \mathbf{w}\left(\phi_{v_{1}}(\tau)+\theta\left(\phi_{v_{\delta}}(\tau)-\phi_{v_{1}}(\tau)\right), x\right)\left(\phi_{v_{\delta}}(\tau)-\phi_{v_{1}}(\tau)\right) \cdot \nabla u_{v_{1}}^{i}(\tau, x) \mathrm{d} \theta
\end{aligned}
$$

$$
\begin{gathered}
\frac{v_{\delta}(\tau)}{\delta} \nabla \cdot\left(\mu_{i} \nabla u_{v_{\delta}}^{i}(\tau, x)\right)-\frac{v_{1}(\tau)}{\delta} \nabla \cdot\left(\mu_{i} \nabla u_{v_{1}}^{i}(\tau, x)\right)=\left(v_{2}(\tau)-v_{1}(\tau)\right) \nabla \cdot\left(\mu_{i} \nabla u_{v_{1}}^{i}(\tau, x)\right) \\
+\frac{v_{\delta}(\tau)}{\delta} \nabla \cdot\left(\mu_{i} \nabla\left(u_{v_{\delta}}^{i}-u_{v_{1}}^{i}\right)(\tau, x)\right) .
\end{gathered}
$$

We also define the following terms, for $i=1, \ldots, 4$ :

$$
\begin{aligned}
\mathbf{A}_{\delta}(\tau, x) & =\int_{0}^{1} D_{\mathbf{u}} \mathbf{A}\left(\phi_{v_{\delta}}(\tau), x, \mathbf{u}_{v_{1}}(\tau, x)+\theta\left(\mathbf{u}_{v_{\delta}}(\tau, x)-\mathbf{u}_{v_{1}}(\tau, x)\right)\right) \mathrm{d} \theta \\
f_{\delta}^{i}(\tau, x)= & \left\{v_{\delta}(\tau) \int_{0}^{1} \nabla_{\mathbf{u}} A^{i}\left(\phi_{v_{\delta}}(\tau), x, \mathbf{u}_{v_{1}}(\tau, x)+\theta\left(\mathbf{u}_{v_{\delta}}(\tau, x)-\mathbf{u}_{v_{1}}(\tau, x)\right)\right) \mathrm{d} \theta\right. \\
& \left.-v_{1}(\tau) \nabla_{\mathbf{u}} A^{i}\left(\phi_{v_{1}}(\tau), x, \mathbf{u}_{v_{1}}(\tau, x)\right)\right\} \cdot \mathbf{h}(\tau, x) \\
& +\left\{v_{\delta}(\tau) \int_{0}^{1} \frac{\partial A^{i}}{\partial t}\left(\phi_{v_{1}}(\tau)+\theta\left(\phi_{v_{\delta}}(\tau)-\phi_{v_{1}}(\tau)\right), x, \mathbf{u}_{v_{1}}(\tau, x)\right) \mathrm{d} \theta\right. \\
& \left.-v_{1}(\tau) \frac{\partial A^{i}}{\partial t}\left(\phi_{v_{1}}(\tau), x, \mathbf{u}_{v_{1}}(\tau, x)\right)\right\} \frac{\phi_{v_{\delta}}(\tau)-\phi_{v_{1}}(\tau)}{\delta} \\
& -\left\{v_{\delta}(\tau) \int_{0}^{1} D_{t} \mathbf{w}\left(\phi_{v_{1}}(\tau)+\theta\left(\phi_{v_{\delta}}(\tau)-\phi_{v_{1}}(\tau)\right), x\right) \cdot \nabla u_{v_{1}}^{i}(\tau, x) \mathrm{d} \theta\right. \\
& \left.-v_{1}(\tau) D_{t} \mathbf{w}\left(\phi_{v_{1}}(\tau), x\right) \cdot \nabla u_{v_{1}}^{i}(\tau, x)\right\} \frac{\phi_{v_{\delta}}(\tau)-\phi_{v_{1}}(\tau)}{\delta} \\
& -\left(v_{\delta}(\tau) \mathbf{w}_{i}\left(\phi_{v_{\delta}}(\tau), x\right)-v_{1}(\tau) \mathbf{w}_{i}\left(\phi_{v_{1}}(\tau), x\right)\right) \cdot \nabla h^{i}(\tau, x) \\
& +\left(v_{\delta}(\tau)-v_{1}(\tau)\right) \nabla \cdot\left(\mu_{i} \nabla h^{i}(\tau, x)\right) .
\end{aligned}
$$

Thus, from equalities (4.22)-(4.24), taking into account that $\frac{u_{v_{\delta}}^{i}-u_{v_{1}}^{i}}{\delta}-h^{i}=z_{\delta}^{i}$ and $\frac{\phi_{v_{\delta}}-\phi_{v_{1}}}{\delta}=\phi_{v_{2}}-\phi_{v_{1}}$, and using notations (4.25)-(4.26), we have that above system (4.19), satisfied by $\mathbf{z}_{\delta}$, can be rewritten in the following 
equivalent way, for $i=1, \ldots, 4$ :

$$
\begin{cases}\frac{\partial z_{\delta}^{i}}{\partial \tau}+v_{\delta}(\tau) \mathbf{w}_{i}\left(\phi_{\delta}(\tau), x\right) \cdot \nabla z_{\delta}^{i}-v_{\delta}(\tau) \nabla \cdot\left(\mu_{i} \nabla z_{\delta}^{i}\right) & \\ =v_{\delta}(\tau)\left[\mathbf{A}_{\delta}(\tau, x) \mathbf{z}_{\delta}\right]_{i}+f_{\delta}^{i}(\tau, x) & \\ \frac{\partial z_{\delta}^{i}}{\partial n}=0 & \text { in }(0,1) \times \Omega \\ z_{\delta}^{i}(0)=0 & \text { in } \Omega .\end{cases}
$$

Now, thanks to estimate (4.12), we have:

$$
\left\|\mathbf{A}_{\delta}\right\|_{L^{\infty}((0,1) \times \Omega)^{4 \times 4}} \leq C, \quad \forall \delta \in(0,1)
$$

Then, choosing as test function $\mathbf{z}_{\delta}$ in system (4.27), integrating by parts and taking into account the boundedness of $v_{\delta}$ and $\mathbf{A}_{\delta}$, we obtain a.e. $\tau \in(0,1)$ :

$$
\left\|\mathbf{z}_{\delta}(\tau)\right\|_{\mathbf{H}}^{2}+2 \mu \int_{0}^{\tau} v_{\delta}(s) \int_{\Omega} \nabla \mathbf{z}_{\delta}: \nabla \mathbf{z}_{\delta} \mathrm{d} x \mathrm{~d} s \leq C \int_{0}^{\tau}\left\|\mathbf{z}_{\delta}(s)\right\|_{\mathbf{H}}^{2} \mathrm{~d} s+\int_{0}^{\tau}\left\|\mathbf{f}_{\delta}(s)\right\|_{\mathbf{H}}^{2} \mathrm{~d} s,
$$

with $\mu=\min \left\{\mu_{1}, \ldots, \mu_{4}\right\}$. Then, by Gronwall's Lemma, we obtain that:

$$
\left\|\mathbf{z}_{\delta}\right\|_{W^{1,2,2}\left(0,1 ; \mathbf{V}, \mathbf{V}^{\prime}\right)} \leq C\left\|\mathbf{f}_{\delta}\right\|_{L^{2}((0,1) \times \Omega)} .
$$

On the other hand, thanks to estimates (4.12) and (4.13), by the dominated convergence Lebesgue's Theorem, we deduce that:

$$
\lim _{\delta \rightarrow 0}\left\|\mathbf{f}_{\delta}\right\|_{\left.L^{2}((0,1) \times \Omega)\right)}=0
$$

Thus, we conclude the convergence of $\mathbf{z}_{\delta}$ to 0 or, equivalently, the desired convergence (4.18).

By completely analogous arguments we can also prove the following result for the differentiability of the state system with respect to the distributed control variable $\varrho$ :

Theorem 4.5. We consider, for any given $C>0$, the following closed, convex subset of $\left.L^{2}((0,1) \times \Omega)\right)$ :

$$
\Upsilon_{C}=\left\{\varrho \in L^{2}((0,1) \times \Omega): 0 \leq \varrho(\tau, x) \leq C \quad \text { a.e. } \quad(\tau, x) \in(0,1) \times \Omega\right\}
$$

and define, $\forall \varrho \in \Upsilon_{C}$, the element $\mathbf{u}_{\varrho} \in W^{1,2,2}\left(0,1 ; \mathbf{V}, \mathbf{V}^{\prime}\right) \cap\left[L^{\infty}((0,1) \times \Omega)\right]^{4}$ as the solution of the system (4.15), where initial condition $\mathbf{u}_{0} \in\left[L^{\infty}(\Omega)\right]^{4}$ is such that $0 \leq u_{0}^{i}(x) \leq M$ a.e. $x \in \Omega, i=1, \ldots, 4$, water velocity $\mathbf{w}$ and temperature $\theta$ satisfy hypothesis $(3.2)$, mapping $v \in \mathcal{C}\left([0,1] ; \mathbb{R}_{*}^{+}\right)$, and $\phi_{v} \in \mathcal{C}^{1}([0,1] ; \mathbb{R})$ is the solution of the initial value problem (4.16).

If $\forall \varrho_{1}, \varrho_{2} \in \Upsilon_{C}$ and $\forall \delta \in(0,1)$ we denote $\varrho_{\delta}=\varrho_{1}+\delta\left(\varrho_{2}-\varrho_{1}\right)$, then we have that $\mathbf{u}_{\varrho_{\delta}}$ verifies the equality:

$$
\mathbf{u}_{\varrho \delta}=\mathbf{u}_{\varrho_{1}}+\delta \mathbf{h}+\mathbf{r}_{\delta}
$$

where $\mathbf{h} \in W^{1,2,2}\left(0,1 ; \mathbf{V}, \mathbf{V}^{\prime}\right)$ is the solution of the system, for $i=1, \ldots, 4$ :

$$
\begin{cases}\frac{\partial h^{i}}{\partial t}+v(\tau) \mathbf{w}_{i}\left(\phi_{v}(\tau), x\right) \cdot \nabla h^{i}-v(\tau) \nabla \cdot\left(\mu_{i} \nabla h^{i}\right) & \\ \quad=v(\tau)\left[D_{\mathbf{u}} \mathbf{A}\left(\phi_{v}(\tau), x, \mathbf{u}_{\rho_{1}}\right) \mathbf{h}\right]_{i}+\delta_{2 i} v(\tau)\left(\varrho_{2}-\varrho_{1}\right) & \text { in }(0,1) \times \Omega, \\ \frac{\partial h^{i}}{\partial n}=0 & \text { on }(0,1) \times \partial \Omega, \\ h^{i}(0)=0 & \text { in } \Omega,\end{cases}
$$


and where $\mathbf{r}_{\delta} \in W^{1,2,2}\left(0,1 ; \mathbf{V}, \mathbf{V}^{\prime}\right)$ is such that:

$$
\lim _{\delta \rightarrow 0} \frac{1}{\delta}\left\|\mathbf{r}_{\delta}\right\|_{W^{1,2,2}\left(0,1 ; \mathbf{V}, \mathbf{V}^{\prime}\right)}=0 .
$$

We must remark here that above result remains true under the milder hypothesis (3.1) with the only difference that $\mathbf{u}_{\varrho}, \mathbf{h}$ and $\mathbf{r}_{\delta} \in W^{1,2,1}\left(0,1 ; \mathbf{V}, \mathbf{V}^{\prime}\right)$. In the proof, since $\mathbf{z}_{\delta} \in L^{2}(0,1 ; \mathbf{V})$ but $\frac{\partial \mathbf{z}_{\delta}}{\partial t} \in L^{1}\left(0,1 ; \mathbf{V}^{\prime}\right)$, we can not use $\mathbf{z}_{\delta}$ as test function in the derivation of the energy estimate for $\mathbf{z}_{\delta}(\tau)$. So, we will need to use, instead of it, a truncate of $\mathbf{z}_{\delta}$ (now in $L^{\infty}(0,1 ; \mathbf{V})$ ) as test function, following the same arguments as those previously introduced by the authors in [2].

Corollary 4.6. Let $\mathbf{u}_{0} \in\left[L^{\infty}(\Omega)\right]^{4}$ be such that $0 \leq u_{0}^{i}(x) \leq M$ a.e. $x \in \Omega, i=1, \ldots, 4$, and let $\mathbf{w}$ and $\theta$ satisfy hypothesis (3.2). Then, for any given $0<C_{1}<C_{2}$ and $C>0$, the mapping

$$
\begin{aligned}
\mathbf{S}: \Psi_{C_{1}, C_{2}} \times \Upsilon_{C} & \longrightarrow W^{1,2,2}\left(0,1 ; \mathbf{V}, \mathbf{V}^{\prime}\right) \\
(v, \varrho) & \longrightarrow \mathbf{S}(v, \varrho)=\mathbf{u}_{v, \varrho}
\end{aligned}
$$

where $\mathbf{u}_{v, \varrho} \in W^{1,2,2}\left(0,1 ; \mathbf{V}, \mathbf{V}^{\prime}\right)$ is the solution of system (4.15), with $\phi_{v} \in \mathcal{C}^{1}([0,1] ; \mathbb{R})$ the solution of initial value problem (4.16), is Gateaux differentiable.

Then, as a direct consequence of above results, we obtain the Gateaux differentiability of the mapping control-state for the optimal control problem $(\widetilde{\mathcal{P}})$ :

Theorem 4.7. Let initial condition $\mathbf{u}_{0}^{1} \in\left[L^{\infty}\left(\Omega_{1}\right)\right]^{4}$ be such that $0 \leq u_{0}^{i, 1}(x) \leq M$ a.e. $x \in \Omega_{1}$ and let water velocities $\mathbf{w}^{1}, \mathbf{w}^{2}$ and temperatures $\theta^{1}, \theta^{2}$ be such that satisfy hypothesis (3.2). Let us consider the following mapping:

$$
\begin{aligned}
& \mathbf{T}: \quad \mathcal{W}_{a d} \quad \longrightarrow \quad W^{1,2,2}\left(0,1 ; \mathbf{V}_{1}, \mathbf{V}_{1}^{\prime}\right) \times W^{1,2,2}\left(0,1 ; \mathbf{V}_{2}, \mathbf{V}_{2}^{\prime}\right) \\
& \left(v^{1}, v^{2}, \varrho^{1}, \varrho^{2}\right) \longrightarrow \mathbf{T}\left(v^{1}, v^{2}, \varrho^{1}, \varrho^{2}\right)=\left(\mathbf{T}_{1}\left(v^{1}, v^{2}, \varrho^{1}, \varrho^{2}\right), \mathbf{T}_{2}\left(v^{1}, v^{2}, \varrho^{1}, \varrho^{2}\right)\right)=\left(\mathbf{y}^{1}, \mathbf{y}^{2}\right)
\end{aligned}
$$

where $\left(\mathbf{y}^{1}, \mathbf{y}^{2}\right)$ are the solutions of state systems (4.4)-(4.5) with controls $\left(v^{1}, v^{2}, \varrho^{1}, \varrho^{2}\right)$. Then, $\mathbf{T}$ is Gateaux differentiable. Moreover, $\forall\left(\widetilde{v}^{1}, \widetilde{v}^{2}, \widetilde{\varrho}^{1}, \widetilde{\varrho}^{2}\right),\left(v^{1}, v^{2}, \varrho^{1}, \varrho^{2}\right) \in \mathcal{W}_{\text {ad }}$ the corresponding derivatives are given by:

$D_{\varrho^{1}} \mathbf{T}_{1}\left(\widetilde{v}^{1}, \widetilde{v}^{2}, \widetilde{\varrho}^{1}, \widetilde{\varrho}^{2}\right)\left(\varrho^{1}-\widetilde{\varrho}^{1}\right)=\mathbf{v}_{\varrho^{1}}^{1} \in W^{1,2,2}\left(0,1 ; \mathbf{V}_{1}, \mathbf{V}_{1}^{\prime}\right) \cap\left[L^{\infty}\left((0,1) \times \Omega_{1}\right)\right]^{4}$ is the solution of system:

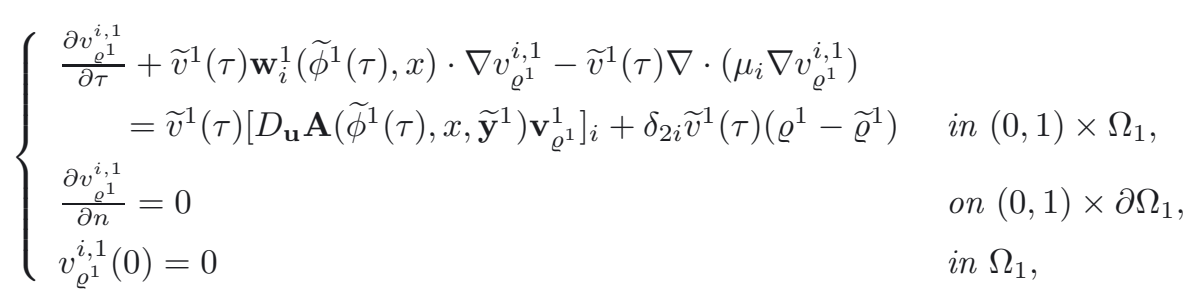

where $\widetilde{\mathbf{y}}^{j}=\mathbf{T}_{j}\left(\widetilde{v}^{1}, \widetilde{v}^{2}, \widetilde{\varrho}^{1}, \widetilde{\varrho}^{2}\right), j=1,2$, and $\widetilde{\phi}^{j}$ are the changes of variable $\widetilde{\phi}^{j}(\tau)=\int_{0}^{\tau} \widetilde{v}^{j}(\zeta) \mathrm{d} \zeta, j=i, 2$.

$D_{\varrho^{2}} \mathbf{T}_{1}\left(\widetilde{v}^{1}, \widetilde{v}^{2}, \widetilde{\varrho}^{1}, \widetilde{\varrho}^{2}\right)\left(\varrho^{2}-\widetilde{\varrho}^{2}\right)=\mathbf{v}_{\varrho^{2}}^{1}=0$.

$D_{\varrho^{1}} \mathbf{T}_{2}\left(\widetilde{v}^{1}, \widetilde{v}^{2}, \widetilde{\varrho}^{1}, \widetilde{\varrho}^{2}\right)\left(\varrho^{1}-\widetilde{\varrho}^{1}\right)=\mathbf{v}_{\varrho^{1}}^{2} \in W^{1,2,2}\left(0,1 ; \mathbf{V}_{2}, \mathbf{V}_{2}^{\prime}\right) \cap\left[L^{\infty}\left((0,1) \times \Omega_{2}\right)\right]^{4}$ is the solution of system:

$$
\left\{\begin{array}{rlrl}
\frac{\partial v_{\rho^{1}}^{i, 2}}{\partial \tau}+\widetilde{v}^{2}(\tau) \mathbf{w}_{i}^{2}\left(\widetilde{\phi}^{2}(\tau), x\right) \cdot \nabla v_{\varrho^{1}}^{i, 2}-\widetilde{v}^{2}(\tau) \nabla \cdot\left(\mu_{i} \nabla v_{\varrho^{1}}^{i, 2}\right) & \\
\quad=\widetilde{v}^{2}(\tau)\left[D_{\mathbf{u}} \mathbf{A}\left(\widetilde{\phi}^{2}(\tau), x, \widetilde{\mathbf{y}}^{2}\right) \mathbf{v}_{\varrho^{1}}^{2}\right]_{i} & & \text { in }(0,1) \times \Omega_{2}, \\
\frac{\partial v_{\rho^{1}}^{i, 2}}{\partial n}=0 & & \text { on }(0,1) \times \partial \Omega_{2}, \\
v_{\varrho^{1}}^{i, 2}(0)=\frac{1}{\operatorname{meas}\left(\Omega_{1}\right)} M_{1}^{i}\left(\mathbf{v}_{\varrho^{1}}^{1}(1)\right) & \text { in } \Omega_{2} .
\end{array}\right.
$$


$D_{\varrho^{2}} \mathbf{T}_{2}\left(\widetilde{v}^{1}, \widetilde{v}^{2}, \widetilde{\varrho}^{1}, \widetilde{\varrho}^{2}\right)\left(\varrho^{2}-\widetilde{\varrho}^{2}\right)=\mathbf{v}_{\varrho^{2}}^{2} \in W^{1,2,2}\left(0,1 ; \mathbf{V}_{2}, \mathbf{V}_{2}^{\prime}\right) \cap\left[L^{\infty}\left((0,1) \times \Omega_{2}\right)\right]^{4}$ is the solution of system:

$$
\begin{cases}\frac{\partial v_{\varrho^{2}}^{i, 2}}{\partial \tau}+\widetilde{v}^{2}(\tau) \mathbf{w}_{i}^{2}\left(\widetilde{\phi}^{2}(\tau), x\right) \cdot \nabla v_{\varrho^{2}}^{i, 2}-\widetilde{v}^{2}(\tau) \nabla \cdot\left(\mu_{i} \nabla v_{\varrho^{2}}^{i, 2}\right) & \\ \quad=\widetilde{v}^{2}(\tau)\left[D_{\mathbf{u}} \mathbf{A}\left(\widetilde{\phi}^{2}(\tau), x, \widetilde{\mathbf{y}}^{2}\right) \mathbf{v}_{\varrho^{2}}^{2}\right]_{i}+\delta_{2 i} \widetilde{v}^{2}(\tau)\left(\varrho^{2}-\widetilde{\varrho}^{2}\right) & \text { in }(0,1) \times \Omega_{2}, \\ \frac{\partial v_{\rho^{2}}^{i, 2}}{\partial n}=0 & \text { on }(0,1) \times \partial \Omega_{2}, \\ v_{\varrho^{2}}^{i, 2}(0)=0 & \text { in } \Omega_{2} .\end{cases}
$$

$D_{v^{1}} \mathbf{T}_{1}\left(\widetilde{v}^{1}, \widetilde{v}^{2}, \widetilde{\varrho}^{1}, \widetilde{\varrho}^{2}\right)\left(v^{1}-\widetilde{v}^{1}\right)=\mathbf{v}_{v^{1}}^{1} \in W^{1,2,2}\left(0,1 ; \mathbf{V}_{1}, \mathbf{V}_{1}^{\prime}\right)$ is the solution of system:

$$
\begin{cases}\frac{\partial v_{v 1}^{i, 1}}{\partial \tau}+\widetilde{v}^{1}(\tau) \mathbf{w}_{i}^{1}\left(\widetilde{\phi}^{1}(\tau), x\right) \cdot \nabla v_{v^{1}}^{i, 1}-\widetilde{v}^{1}(\tau) \nabla \cdot\left(\mu_{i} \nabla v_{v^{1}}^{i, 1}\right)=\widetilde{v}^{1}(\tau)\left[D_{\mathbf{u}} \mathbf{A}\left(\widetilde{\phi}^{1}(\tau), x, \widetilde{\mathbf{y}}^{1}\right) \mathbf{v}_{v^{1}}^{1}\right]_{i} & \\ \quad+\left(\phi^{1}(\tau)-\widetilde{\phi}^{1}(\tau)\right)\left[\widetilde{v}^{1}(\tau) D_{t} A^{i}\left(\widetilde{\phi}^{1}(\tau), x, \widetilde{\mathbf{y}}^{1}\right)-\widetilde{v}^{1}(\tau) D_{t} \mathbf{w}^{1}\left(\widetilde{\phi}^{1}(\tau), x\right) \cdot \nabla \widetilde{y}^{i, 1}\right] & \\ \quad+\left(v^{1}(\tau)-\widetilde{v}^{1}(\tau)\right)\left[\nabla \cdot\left(\mu_{i} \nabla \widetilde{y}^{i, 1}\right)-\mathbf{w}_{i}^{1}\left(\widetilde{\phi}^{1}(\tau), x\right) \cdot \nabla \widetilde{y}^{i, 1}+A^{i}\left(\widetilde{\phi}^{1}(\tau), x, \widetilde{\mathbf{y}}^{1}\right)+\delta_{2 i} \widetilde{\varrho}^{1}\right] & \text { in }(0,1) \times \Omega_{1}, \\ \frac{\partial v_{v 1}^{i, 1}}{\partial n}=0 & \text { on }(0,1) \times \partial \Omega_{1}, \\ v_{v^{1}}^{i, 1}(0)=0 & \text { in } \Omega_{1},\end{cases}
$$

where $\phi^{1} \in \mathcal{C}^{1}([0,1] ; \mathbb{R})$ is the solution of the initial value problem (4.6).

$D_{v^{2}} \mathbf{T}_{1}\left(\widetilde{v}^{1}, \widetilde{v}^{2}, \widetilde{\varrho}^{1}, \widetilde{\varrho}^{2}\right)\left(v^{2}-\widetilde{v}^{2}\right)=\mathbf{v}_{v^{2}}^{1}=0$.

$D_{v^{1}} \mathbf{T}_{2}\left(\widetilde{v}^{1}, \widetilde{v}^{2}, \widetilde{\varrho}^{1}, \widetilde{\varrho}^{2}\right)\left(v^{1}-\widetilde{v}^{1}\right)=\mathbf{v}_{v^{1}}^{2} \in W^{1,2,2}\left(0,1 ; \mathbf{V}_{2}, \mathbf{V}_{2}^{\prime}\right)$ is the solution of system:

$$
\left\{\begin{array}{rlrl}
\frac{\partial v_{v 1}^{i, 2}}{\partial \tau}+\widetilde{v}^{2}(\tau) \mathbf{w}_{i}^{2}\left(\widetilde{\phi}^{2}(\tau), x\right) \cdot \nabla v_{v^{1}}^{i, 2}-\widetilde{v}^{2}(\tau) \nabla \cdot\left(\mu_{i} \nabla v_{v^{1}}^{i, 2}\right) & \\
=\widetilde{v}^{2}(\tau)\left[D_{\mathbf{u}} \mathbf{A}\left(\widetilde{\phi}^{2}(\tau), x, \widetilde{\mathbf{y}}^{2}\right) \mathbf{v}_{v^{1}}^{2}\right]_{i} & & \text { in }(0,1) \times \Omega_{2}, \\
\frac{\partial v_{v 1}^{i, 1}}{\partial n}=0 & \text { on }(0,1) \times \partial \Omega_{2}, \\
v_{v^{1}}^{i, 1}(0)=\frac{1}{\operatorname{meas}\left(\Omega_{1}\right)} M_{1}^{i}\left(\mathbf{v}_{v^{1}}^{1}(1)\right) & \text { in } \Omega_{2} .
\end{array}\right.
$$

$D_{v^{2}} \mathbf{T}_{2}\left(\widetilde{v}^{1}, \widetilde{v}^{2}, \widetilde{\varrho}^{1}, \widetilde{\varrho}^{2}\right)\left(v^{2}-\widetilde{v}^{2}\right)=\mathbf{v}_{v^{2}}^{2} \in W^{1,2,2}\left(0,1 ; \mathbf{V}_{2}, \mathbf{V}_{2}^{\prime}\right)$ is the solution of system:

$$
\begin{cases}\frac{\partial v_{v 2}^{i, 2}}{\partial \tau}+\widetilde{v}^{2}(\tau) \mathbf{w}_{i}^{2}\left(\widetilde{\phi}^{2}(\tau), x\right) \cdot \nabla v_{v^{2}}^{i, 2}-\widetilde{v}^{2}(\tau) \nabla \cdot\left(\mu_{i} \nabla v_{v^{2}}^{i, 2}\right)=\widetilde{v}^{2}(\tau)\left[D_{\mathbf{u}} \mathbf{A}\left(\widetilde{\phi}^{2}(\tau), x, \widetilde{\mathbf{y}}^{2}\right) \mathbf{v}_{v^{2}}^{2}\right]_{i} & \\ \quad+\left(\phi^{2}(\tau)-\widetilde{\phi}^{2}(\tau)\right)\left[\widetilde{v}^{2}(\tau) D_{t} A^{i}\left(\widetilde{\phi}^{2}(\tau), x, \widetilde{\mathbf{y}}^{2}\right)-\widetilde{v}^{2}(\tau) D_{t} \mathbf{w}^{2}\left(\widetilde{\phi}^{2}(\tau), x\right) \cdot \nabla \widetilde{y}^{i, 2}\right] & \\ \quad+\left(v^{2}(\tau)-\widetilde{v}^{2}(\tau)\right)\left[\nabla \cdot\left(\mu_{i} \nabla \widetilde{y}^{i, 2}\right)-\mathbf{w}_{i}^{2}\left(\widetilde{\phi}^{2}(\tau), x\right) \cdot \nabla \widetilde{y}^{i, 2}+A^{i}\left(\widetilde{\phi}^{2}(\tau), x, \widetilde{\mathbf{y}}^{2}\right)+\delta_{2 i} \widetilde{\varrho}^{2}\right] & \text { in }(0,1) \times \Omega_{2}, \\ \frac{\partial v_{v 2}^{i, 2}}{n n}=0 & \text { on }(0,1) \times \partial \Omega_{2}, \\ v_{v^{2}}^{i, 2}(0)=0 & \text { in } \Omega_{2},\end{cases}
$$

where $\phi^{2} \in \mathcal{C}^{1}([0,1] ; \mathbb{R})$ is the solution of the initial value problem $(4.7)$.

First order optimality conditions for state constrained optimal control problems can be obtained by different techniques. However, in, our particular case, and due to its special features, we will derive optimality conditions 
for optimal control problem $(\widetilde{\mathcal{P}})$ by using the following abstract result of Casas [8], whose application to our problem is immediate:

Theorem 4.8. Let us consider the following optimization problem:

$$
\inf _{\mathbf{b} \in U_{a d}} F(\mathbf{b}) \quad \text { subject to } G(\mathbf{b}) \in E,
$$

where $U_{a d}$ and $E$ are, respectively, two convex subsets of two Banach spaces $V$ and $W$, satisfying $\stackrel{\circ}{E} \neq \emptyset$, and $F: V \longrightarrow(-\infty,+\infty]$ and $G: V \longrightarrow W$ are two Gateaux differentiable mappings. Then, if $\widetilde{\mathbf{b}} \in U_{a d}$ is a solution of problem $(\mathcal{Q})$, there will exist elements $\gamma \geq 0$ and $\lambda \in W^{\prime}$ such that:

$$
\begin{aligned}
& \gamma+\|\lambda\|_{W^{\prime}}>0, \\
& \langle\lambda, \beta-G(\widetilde{\mathbf{b}})\rangle_{W^{\prime}, W} \leq 0, \quad \forall \beta \in E, \\
& \left\langle\gamma F^{\prime}(\widetilde{\mathbf{b}})+\left[\frac{D G}{D \mathbf{b}}(\widetilde{\mathbf{b}})\right]^{*} \lambda, \mathbf{b}-\widetilde{\mathbf{b}}\right\rangle_{V^{\prime}, V} \geq 0, \quad \forall \mathbf{b} \in \mathcal{U}_{a d} .
\end{aligned}
$$

Moreover, if the problem $(\mathcal{Q})$ is qualified, that is, if there exists an element $\mathbf{b}_{0} \in U_{\text {ad }}$ satisfying:

$$
G(\tilde{\mathbf{b}})+\frac{D G}{D \mathbf{b}}(\tilde{\mathbf{b}})\left(\mathbf{b}_{0}-\tilde{\mathbf{b}}\right) \in \stackrel{\circ}{E}
$$

then we obtain above relations for $\gamma>0$.

In order to simplify the notation in below paragraphs we will introduce the following functionals $\mathcal{H}^{j}$ : $\mathbb{R}_{*}^{+} \times\left\{\mathbf{u} \in \mathbf{V}_{j}: u^{i}(x) \geq 0\right.$ a.e. $\left.x \in \Omega_{j}\right\} \times \mathbf{V}_{j} \times L^{2}\left(\Omega_{j}\right) \times \mathbb{R} \rightarrow \mathbb{R}, j=1,2$, define as:

$$
\begin{aligned}
\mathcal{H}^{j}(t, \mathbf{u}, \mathbf{p}, \rho, \gamma)=\gamma N_{1}+\int_{\Omega_{j}} \mathbf{A}(t, x, \mathbf{u}(x)) \cdot \mathbf{p}(x) \mathrm{d} x+\int_{\Omega_{j}} p^{2}(x) \rho(x) \mathrm{d} x-\int_{\Omega_{j}} \Lambda_{\mu} \nabla \mathbf{u}(x): & \quad \nabla \mathbf{p}(x) \mathrm{d} x-\int_{\Omega_{j}} \nabla \mathbf{u}(x) \mathbf{w}^{j}(t, x) \cdot \mathbf{p}(x) \mathrm{d} x,
\end{aligned}
$$

where $\Lambda_{\mu}$ is the diagonal matrix whose diagonal is given by $\left(\mu_{1}, \mu_{2}, \mu_{3}, \mu_{4}\right)$.

Then, as a consequence of the adaptation of above Theorem 4.8 to our fixed final time problem $(\widetilde{\mathcal{P}})$, we have the following results providing us with first order optimality conditions for the problem:

Theorem 4.9. Let initial condition $\mathbf{u}_{0}^{1} \in\left[L^{\infty}\left(\Omega_{1}\right)\right]^{4}$ be such that $0 \leq u_{0}^{i, 1}(x) \leq M$ a.e. $x \in \Omega_{1}$ and let water velocities $\mathbf{w}^{1}, \mathbf{w}^{2}$ and temperatures $\theta^{1}, \theta^{2}$ be such that satisfy hypothesis $(3.2)$. Let $\left(\widetilde{v}^{1}, \widetilde{v}^{2}, \widetilde{\varrho}^{1}, \widetilde{\varrho}^{2}\right) \in \mathcal{W}_{\text {ad }}$ be a solution of the optimal control problem $(\widetilde{\mathcal{P}})$ with associated state $\left(\widetilde{\mathbf{y}}^{1}, \widetilde{\mathbf{y}}^{2}, \widetilde{\phi}^{1}, \widetilde{\phi}^{2}\right) \in\left(W^{1,2,2}\left(0,1 ; \mathbf{V}_{1}, \mathbf{V}_{1}^{\prime}\right) \cap\right.$ $\left.\left[L^{\infty}\left((0,1) \times \Omega_{1}\right)\right]^{4}\right) \times\left(W^{1,2,2}\left(0,1 ; \mathbf{V}_{2}, \mathbf{V}_{2}^{\prime}\right) \cap\left[L^{\infty}\left((0,1) \times \Omega_{2}\right)\right]^{4}\right) \times \mathcal{C}^{1}([0,1] ; \mathbb{R})^{2}$. Then, there exist elements $\gamma \geq 0$ and $\lambda=\left(\lambda_{1}, \lambda_{2}, \lambda_{3}, \lambda_{4}\right) \in \mathbb{R}^{4}$, with $(\gamma, \lambda) \neq 0 \in \mathbb{R}^{5}$, such that:

$$
\begin{gathered}
\left\langle\lambda, \widetilde{\mathbf{B}}\left(\widetilde{v}^{1}, \widetilde{v}^{2}, \widetilde{\varrho}^{1}, \widetilde{\varrho}^{2}\right)-\beta\right\rangle_{\mathbb{R}^{4}} \geq 0, \quad \forall \beta \in\left[0, \sigma_{1}\right] \times\left[0, \sigma_{2}\right] \times\left[\theta_{1}, \infty\right) \times\left[\theta_{2}, \infty\right), \\
\sum_{j=1}^{2} \int_{(0,1) \times \Omega_{j}} \widetilde{v}^{j}\left(\varrho^{j}-\widetilde{\varrho}^{j}\right) q^{2, j} \mathrm{~d} x \mathrm{~d} \tau \geq 0, \quad \forall\left(\varrho^{1}, \varrho^{2}\right) \in \mathcal{W}_{a d}^{2}, \\
\sum_{j=1}^{2} \int_{0}^{1}\left(v^{j}(\tau)-\widetilde{v}^{j}(\tau)\right)\left[r^{j}(\tau)+\mathcal{H}^{j}\left(\widetilde{\phi}^{j}(\tau), \widetilde{\mathbf{y}}^{j}(\tau), \mathbf{q}^{j}(\tau), \widetilde{\varrho}^{j}(\tau), \gamma\right)\right] \mathrm{d} \tau \geq 0, \quad \forall\left(v^{1}, v^{2}\right) \in \mathcal{W}_{a d}^{1},
\end{gathered}
$$


where $\left(\mathbf{q}^{1}, \mathbf{q}^{2}\right) \in\left(W^{1,2,2}\left(0,1 ; \mathbf{V}_{1}, \mathbf{V}_{1}^{\prime}\right) \cap\left[L^{\infty}\left((0,1) \times \Omega_{1}\right)\right]^{4}\right) \times\left(W^{1,2,2}\left(0,1 ; \mathbf{V}_{2}, \mathbf{V}_{2}^{\prime}\right) \cap\left[L^{\infty}\left((0,1) \times \Omega_{2}\right)\right]^{4}\right)$ (adjoint states associated to the distributed controls) are the solutions of the following coupled linear adjoint systems, for $i=1, \ldots, 4$ :

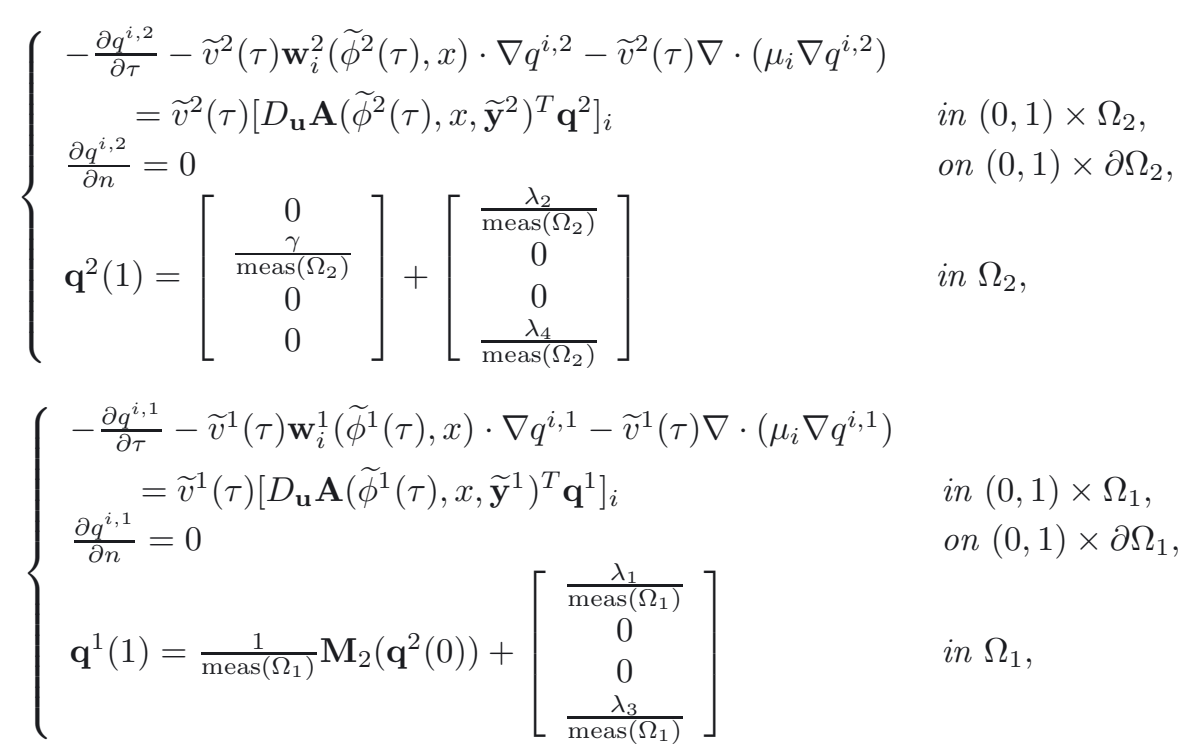

and where $\left(r^{1}, r^{2}\right) \in \mathcal{C}([0,1] ; \mathbb{R})^{2}$ (adjoint states associated to the time controls) are the solutions of the following uncoupled final time problems, for $j=1,2$ :

$$
\left\{\begin{array}{l}
-\frac{\mathrm{d} r^{j}}{\mathrm{~d} \tau}(\tau)=\widetilde{v}^{j}(\tau)\left\langle\mathbf{q}^{j}(\tau), D_{t} \mathbf{A}\left(\widetilde{\phi}^{j}(\tau), x, \widetilde{\mathbf{y}}^{j}(\tau)\right)-D_{t} \mathbf{w}^{j}\left(\widetilde{\phi}^{j}(\tau), x\right) \cdot \nabla \widetilde{\mathbf{y}}^{j}(\tau)\right\rangle_{\mathbf{V}_{j}, \mathbf{V}_{j}^{\prime}} \quad \text { in }(0,1), \\
r^{j}(1)=0 .
\end{array}\right.
$$

Proof. In order to adapt our optimal control problem $(\widetilde{\mathcal{P}})$ to the abstract framework presented in Theorem 4.8, we consider $V=L^{2}\left((0,1) \times \Omega_{1}\right) \times L^{2}\left((0,1) \times \Omega_{2}\right) \times \mathcal{C}\left([0,1] ; \mathbb{R}^{+}\right)^{2}, W=\mathbb{R}^{4}, U_{a d}=\mathcal{W}_{a d}, E=\left[0, \sigma_{1}\right] \times\left[0, \sigma_{2}\right] \times$ $\left[\theta_{1}, \infty\right) \times\left[\theta_{2}, \infty\right) \subset \mathbb{R}^{4}, \mathbf{b}=\left(v^{1}, v^{2}, \varrho^{1}, \varrho^{2}\right), F=\widetilde{J}$, and $G=\widetilde{\mathbf{B}}$. Let $\widetilde{\mathbf{b}}=\left(\widetilde{v}^{1}, \widetilde{v}^{2}, \widetilde{\varrho}^{1}, \widetilde{\varrho}^{2}\right)$ be a solution of the problem $(\widetilde{\mathcal{P}})$. Then, thanks to Theorem 4.8, we obtain the existence of elements $\gamma \geq 0$ and $\lambda \in \mathbb{R}^{4}$, with $\gamma+\|\lambda\|_{\mathbb{R}^{4}}>0$, such that:

$$
\langle\lambda, \beta-G(\widetilde{\mathbf{b}})\rangle_{\mathbb{R}^{4}} \leq 0, \quad \forall \beta \in E,
$$

and

$$
\left\langle\gamma F^{\prime}(\widetilde{\mathbf{b}})+\left[\frac{D G}{D \mathbf{b}}(\widetilde{\mathbf{b}})\right]^{*} \lambda, \mathbf{b}-\widetilde{\mathbf{b}}\right\rangle_{V^{\prime}, V}=\gamma F^{\prime}(\widetilde{\mathbf{b}})(\mathbf{b}-\widetilde{\mathbf{b}})+\left\langle\lambda, \frac{D G}{D \mathbf{b}}(\widetilde{\mathbf{b}})(\mathbf{b}-\widetilde{\mathbf{b}})\right\rangle_{\mathbb{R}^{4}} \geq 0, \quad \forall \mathbf{b} \in \mathcal{U}_{a d}
$$

On one hand, it is clear that inequality (4.48) is equivalent to condition (4.42). On the other hand, taking into account the notations and results from Theorem 4.7, we have that:

$$
\begin{aligned}
\left\langle\lambda, \frac{D G}{D \mathbf{b}}(\widetilde{\mathbf{b}})(\mathbf{b}-\widetilde{\mathbf{b}})\right\rangle_{\mathbb{R}^{4}}= & \frac{\lambda_{1}}{\operatorname{meas}\left(\Omega_{1}\right)} \int_{\Omega_{1}}\left(v_{\varrho^{1}}^{1,1}(1)+v_{v^{1}}^{1,1}(1)\right) \mathrm{d} x \\
& +\frac{\lambda_{2}}{\operatorname{meas}\left(\Omega_{2}\right)} \int_{\Omega_{2}}\left(v_{\varrho^{1}}^{1,2}(1)+v_{\varrho^{2}}^{1,2}(1)+v_{v^{1}}^{1,2}(1)+v_{v^{2}}^{1,2}(1)\right) \mathrm{d} x \\
& +\frac{\lambda_{3}}{\operatorname{meas}\left(\Omega_{1}\right)} \int_{\Omega_{1}}\left(v_{\varrho^{1}}^{4,1}(1)+v_{v^{1}}^{4,1}(1)\right) \mathrm{d} x \\
& +\frac{\lambda_{4}}{\operatorname{meas}\left(\Omega_{2}\right)} \int_{\Omega_{2}}\left(v_{\varrho^{1}}^{4,2}(1)+v_{\varrho^{2}}^{4,2}(1)+v_{v^{1}}^{4,2}(1)+v_{v^{2}}^{4,2}(1)\right) \mathrm{d} x .
\end{aligned}
$$


From the boundedness and non-negativity of the solutions of the state systems (4.4)-(4.5), we can deduce the existence of unique solutions $\left(\mathbf{q}^{1}, \mathbf{q}^{2}\right) \in\left(W^{1,2,2}\left(0,1 ; \mathbf{V}_{1}, \mathbf{V}_{1}^{\prime}\right) \cap\left[L^{\infty}\left((0,1) \times \Omega_{1}\right)\right]^{4}\right) \times\left(W^{1,2,2}\left(0,1 ; \mathbf{V}_{2}, \mathbf{V}_{2}^{\prime}\right) \cap\right.$ $\left.\left[L^{\infty}\left((0,1) \times \Omega_{2}\right)\right]^{4}\right)$ for the adjoint systems $(4.45)-(4.46)$. Thus, the mapping:

$$
\tau \in(0,1) \rightarrow \widetilde{v}^{j}(\tau)\left\langle\mathbf{q}^{j}(\tau), D_{t} \mathbf{A}\left(\widetilde{\phi}^{j}(\tau), x, \widetilde{\mathbf{y}}^{j}(\tau)\right)-D_{t} \mathbf{w}^{j}\left(\widetilde{\phi}^{j}(\tau), x\right) \cdot \nabla \widetilde{\mathbf{y}}^{j}(\tau)\right\rangle_{\mathbf{V}_{j}, \mathbf{V}_{j}^{\prime}}
$$

lies in space $L^{1}(0,1)$, for $j=1,2$, and, as a direct consequence of classical results for ordinary differential equations, there exists a unique solution $r^{j} \in \mathcal{C}([0,1] ; \mathbb{R})$ for the adjoint equations (4.47). (Actually, $r^{j} \in$ $W^{1,1}(0,1), j=1,2$.)

Taking as test function $\mathbf{z}^{1}=\mathbf{q}^{1}(\tau)$ in (4.32) and $\mathbf{z}^{2}=\mathbf{q}^{2}(\tau)$ in (4.33)-(4.34), integrating along the time interval $(0,1)$, adding obtained expressions, and making use of integration by parts, we obtain:

$$
\begin{array}{r}
\gamma \frac{N_{2}}{\operatorname{meas}\left(\Omega_{2}\right)} \int_{\Omega_{2}}\left(v_{\varrho^{1}}^{2,2}(1)+v_{\varrho^{2}}^{2,2}(1)\right) \mathrm{d} x+\frac{\lambda_{1}}{\operatorname{meas}\left(\Omega_{1}\right)} \int_{\Omega_{1}} v_{\varrho^{1}}^{1,1}(1) \mathrm{d} x+\frac{\lambda_{2}}{\operatorname{meas}\left(\Omega_{2}\right)} \int_{\Omega_{2}}\left(v_{\varrho^{1}}^{1,2}(1)+v_{\varrho^{2}}^{1,2}(1)\right) \mathrm{d} x \\
+\frac{\lambda_{3}}{\operatorname{meas}\left(\Omega_{1}\right)} \int_{\Omega_{1}} v_{\varrho^{1}}^{4,1}(1) \mathrm{d} x+\frac{\lambda_{4}}{\operatorname{meas}\left(\Omega_{2}\right)} \int_{\Omega_{2}}\left(v_{\varrho^{1}}^{4,2}(1)+v_{\varrho^{2}}^{4,2}(1)\right) \mathrm{d} x \\
=\sum_{j=1}^{2} \int_{(0,1) \times \Omega_{j}} \widetilde{v}^{j}\left(\varrho^{j}-\widetilde{\varrho}^{j}\right) q^{2, j} \mathrm{~d} x \mathrm{~d} \tau
\end{array}
$$

Taking now as test function $\mathbf{z}^{1}=\mathbf{q}^{1}(\tau)$ in (4.35) and $\mathbf{z}^{2}=\mathbf{q}^{2}(\tau)$ in (4.36)-(4.37), and integrating again along the time interval $(0,1)$, we also obtain:

$$
\begin{gathered}
\int_{0}^{1}\left(v^{j}(\tau)-\widetilde{v}^{j}(\tau)\right) \int_{\Omega_{j}}\left[\mathbf{A}\left(\widetilde{\phi}^{j}(\tau), x, \widetilde{\mathbf{y}}^{j}(\tau)\right) \cdot \mathbf{q}^{j}(\tau)+\widetilde{\varrho}^{j} q^{2, j}-\Lambda_{\mu} \nabla \widetilde{\mathbf{y}}^{j}(\tau): \nabla \mathbf{q}^{j}(\tau)-\nabla \widetilde{\mathbf{y}}^{j}(\tau) \mathbf{w}^{j}\left(\widetilde{\phi}^{j}(\tau), x\right) \cdot \mathbf{q}^{j}(\tau)\right] \mathrm{d} x \mathrm{~d} \tau \\
=\int_{0}^{1}\left(v^{j}(\tau)-\widetilde{v}^{j}(\tau)\right) \mathcal{H}^{j}\left(\widetilde{\phi}^{j}(\tau), \widetilde{\mathbf{y}}^{j}(\tau), \mathbf{q}^{j}(\tau), \widetilde{\varrho}^{j}(\tau), 0\right) \mathrm{d} \tau \\
\int_{0}^{1}\left(\phi^{j}(\tau)-\widetilde{\phi}^{j}(\tau)\right) \widetilde{v}^{j}(\tau) \int_{\Omega_{j}}\left[D_{t} \mathbf{A}\left(\widetilde{\phi}^{j}(\tau), x, \widetilde{\mathbf{y}}^{j}(\tau)\right) \cdot \mathbf{q}^{j}(\tau)-D_{t} \widetilde{\mathbf{y}}^{j}(\tau) \mathbf{w}^{j}\left(\widetilde{\phi}^{j}(\tau), x\right) \cdot \mathbf{q}^{j}(\tau)\right] \mathrm{d} x \mathrm{~d} \tau \\
=-\int_{0}^{1}\left\langle\frac{\mathrm{d} r^{j}}{\mathrm{~d} \tau}(\tau),\left(\phi^{j}(\tau)-\widetilde{\phi}^{j}(\tau)\right)\right\rangle \mathrm{d} \tau=\int_{0}^{1} r^{j}(\tau)\left(v^{j}(\tau)-\widetilde{v}^{j}(\tau)\right) \mathrm{d} \tau
\end{gathered}
$$

Repeating now above arguments, we obtain:

$$
\begin{aligned}
\gamma N_{1} \sum_{j=1}^{2} \int_{0}^{1}\left(v^{j}(\tau)-\widetilde{v}^{j}(\tau)\right) \mathrm{d} \tau & +\gamma \frac{N_{2}}{\operatorname{meas}\left(\Omega_{2}\right)} \int_{\Omega_{2}}\left(v_{v^{1}}^{2,2}(1)+v_{v^{2}}^{2,2}(1)\right) \\
& +\frac{\lambda_{1}}{\operatorname{meas}\left(\Omega_{1}\right)} \int_{\Omega_{1}} v_{v^{1}}^{1,1}(1) \mathrm{d} x+\frac{\lambda_{2}}{\operatorname{meas}\left(\Omega_{2}\right)} \int_{\Omega_{2}}\left(v_{v^{1}}^{1,2}(1)+v_{v^{2}}^{1,2}(1)\right) \mathrm{d} x \\
& +\frac{\lambda_{3}}{\operatorname{meas}\left(\Omega_{1}\right)} \int_{\Omega_{1}} v_{v^{1}}^{4,1}(1) \mathrm{d} x+\frac{\lambda_{4}}{\operatorname{meas}\left(\Omega_{2}\right)} \int_{\Omega_{2}}\left(v_{v^{1}}^{4,2}(1)+v_{v^{2}}^{4,2}(1)\right) \mathrm{d} x \\
& =\sum_{j=1}^{2} \int_{0}^{1}\left(v^{j}(\tau)-\widetilde{v}^{j}(\tau)\right)\left(r^{j}(\tau)+\mathcal{H}^{j}\left(\widetilde{\phi}^{j}(\tau), \widetilde{\mathbf{y}}^{j}(\tau), \mathbf{q}^{j}(\tau), \widetilde{\varrho}^{j}(\tau), \gamma\right)\right) \mathrm{d} \tau
\end{aligned}
$$


Finally, from equalities (4.51)-(4.54), we have, $\forall \mathbf{b} \in \mathcal{W}_{a d}$, that:

$$
\begin{aligned}
\gamma F^{\prime}(\widetilde{\mathbf{b}})(\mathbf{b}-\widetilde{\mathbf{b}})+\left\langle\lambda, \frac{D G}{D \mathbf{b}}(\widetilde{\mathbf{b}})(\mathbf{b}-\widetilde{\mathbf{b}})\right\rangle_{\mathbb{R}^{4}}=\sum_{j=1}^{2} \int_{(0,1) \times \Omega_{j}} \widetilde{v}^{j}\left(\varrho^{j}-\widetilde{\varrho}^{j}\right) q^{2, j} \mathrm{~d} x \mathrm{~d} \tau \\
\quad+\sum_{j=1}^{2} \int_{0}^{1}\left(v^{j}(\tau)-\widetilde{v}^{j}(\tau)\right)\left[r^{j}(\tau)+\mathcal{H}^{j}\left(\widetilde{\phi}^{j}(\tau), \widetilde{\mathbf{y}}^{j}(\tau), \mathbf{q}^{j}(\tau), \widetilde{\varrho}^{j}(\tau), \gamma\right)\right] \mathrm{d} \tau .
\end{aligned}
$$

Taking this expression to (4.49) we obtain conditions (4.43)-(4.44), which concludes the proof.

\subsection{The original time optimal control problem}

Once we have obtained, in the above subsection, the desired optimality conditions for the auxiliary optimal control problem with fixed final time $(\widetilde{\mathcal{P}})$, we can use them to recover the optimality conditions for our original time optimal control problem $(\mathcal{P})$. So, we have the following results:

Theorem 4.10. Let $\left(\widetilde{T}^{1}, \widetilde{T}^{2}, \widetilde{\rho}^{1}, \widetilde{\rho}^{2}\right) \in \mathcal{V}_{\text {ad }}$ be a solution of the time optimal control problem $(\mathcal{P})$ with associated state $\left(\widetilde{\mathbf{u}}^{1}, \widetilde{\mathbf{u}}^{2}\right) \in\left(W^{1,2,2}\left(0, \widetilde{T}^{1} ; \mathbf{V}_{1}, \mathbf{V}_{1}^{\prime}\right) \cap\left[L^{\infty}\left(\left(0, \widetilde{T}^{1}\right) \times \Omega_{1}\right)\right]^{4}\right) \times\left(W^{1,2,2}\left(0, \widetilde{T}^{2} ; \mathbf{V}_{2}, \mathbf{V}_{2}^{\prime}\right) \cap\left[L^{\infty}\left(\left(0, \widetilde{T}^{2}\right) \times \Omega_{2}\right)\right]^{4}\right)$. Then, there exist elements $\gamma \geq 0$ and $\lambda=\left(\lambda_{1}, \lambda_{2}, \lambda_{3}, \lambda_{4}\right) \in \mathbb{R}^{4}$, with $(\gamma, \lambda) \neq 0 \in \mathbb{R}^{5}$, such that:

$$
\begin{gathered}
\left\langle\lambda, \mathbf{B}\left(\widetilde{T}^{1}, \widetilde{T}^{2}, \widetilde{\rho}^{1}, \widetilde{\rho}^{2}\right)-\beta\right\rangle_{\mathbb{R}^{4}} \geq 0, \quad \forall \beta \in\left[0, \sigma_{1}\right] \times\left[0, \sigma_{2}\right] \times\left[\theta_{1}, \infty\right) \times\left[\theta_{2}, \infty\right), \\
\sum_{j=1}^{2} \int_{\left(0, \widetilde{T}^{j}\right) \times \Omega_{j}}\left(\rho^{j}-\widetilde{\rho}^{j}\right) p^{2, j} \mathrm{~d} x \mathrm{~d} \tau \geq 0, \quad \forall\left(\rho^{1}, \rho^{2}\right) \in \mathcal{V}_{a d}^{2}, \\
\sum_{j=1}^{2}\left(T^{j}-\widetilde{T}^{j}\right)\left[s^{j}\left(t^{j}\right)+\mathcal{H}^{j}\left(t^{j}, \widetilde{\mathbf{u}}^{j}\left(t^{j}\right), \mathbf{p}^{j}\left(t^{j}\right), \widetilde{\rho}^{j}\left(t^{j}\right), \gamma\right)\right] \geq 0 \\
\text { a.e. } \quad t^{j} \in\left(0, \widetilde{T}^{j}\right), j=1,2, \quad \forall\left(T^{1}, T^{2}\right) \in \mathcal{V}_{a d}^{1},
\end{gathered}
$$

where $\left(\mathbf{p}^{1}, \mathbf{p}^{2}\right) \in\left(W^{1,2,2}\left(0, \widetilde{T}^{1} ; \mathbf{V}_{1}, \mathbf{V}_{1}^{\prime}\right) \cap\left[L^{\infty}\left(\left(0, \widetilde{T}^{1}\right) \times \Omega_{1}\right)\right]^{4}\right) \times\left(W^{1,2,2}\left(0, \widetilde{T}^{2} ; \mathbf{V}_{2}, \mathbf{V}_{2}^{\prime}\right) \cap\left[L^{\infty}\left(\left(0, \widetilde{T}^{2}\right) \times \Omega_{2}\right)\right]^{4}\right)$ (adjoint states associated to the distributed controls) are the solutions of the following coupled adjoint systems, for $i=1, \ldots, 4$ :

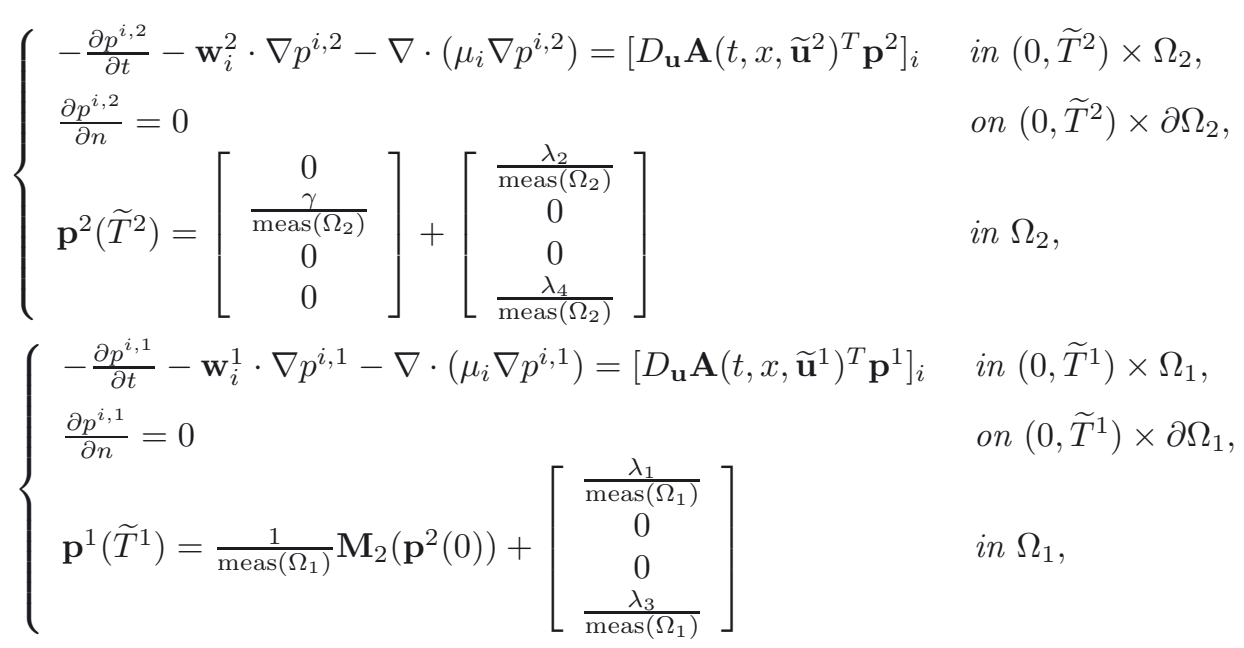


and where $\left(s^{1}, s^{2}\right) \in \mathcal{C}\left(\left[0, \widetilde{T}^{1}\right] ; \mathbb{R}\right) \times \mathcal{C}\left(\left[0, \widetilde{T}^{2}\right] ; \mathbb{R}\right)$ (adjoint states associated to the time controls) are the solutions of the following uncoupled final time problems, for $j=1,2$ :

$$
\left\{\begin{array}{l}
-\frac{\mathrm{d} s^{j}}{\mathrm{~d} t}(t)=\left\langle\mathbf{p}^{j}(t), D_{t} \mathbf{A}\left(t, x, \widetilde{\mathbf{u}}^{j}(t)\right)-D_{t} \mathbf{w}^{j}(t) \cdot \nabla \widetilde{\mathbf{u}}^{j}(t)\right\rangle_{\mathbf{V}_{j}, \mathbf{V}_{j}^{\prime}} \quad \text { in }\left(0, \widetilde{T}^{j}\right), \\
s^{j}\left(\widetilde{T}^{j}\right)=0
\end{array}\right.
$$

Proof. The result will be a consequence of the equivalence of problems $(\mathcal{P})$ and $(\widetilde{\mathcal{P}})$, as proved in Theorem 4.3. So, let us consider a pair of mappings $\left(\widetilde{v}^{1}, \widetilde{v}^{2}\right) \in \mathcal{W}_{a d}^{2}$ such that:

$$
\widetilde{\phi}^{j}(1)=\int_{0}^{1} \widetilde{v}^{j}(\zeta) \mathrm{d} \zeta=\widetilde{T}^{j}, \quad j=1,2
$$

and let $\left(\widetilde{\varrho}^{1}, \widetilde{\varrho}^{2}\right) \in \mathcal{W}_{a d}^{1}$ be the elements given by:

$$
\widetilde{\varrho}^{j}(\tau)=\left(\widetilde{\rho}^{j} \circ \widetilde{\phi}^{j}\right)(\tau), \quad j=1,2 .
$$

Then we have, thanks to Theorem 4.3, that the control $\left(\widetilde{v}^{1}, \widetilde{v}^{2}, \widetilde{\varrho}^{1}, \widetilde{\varrho}^{2}\right) \in \mathcal{W}_{a d}$ is a solution of optimal control problem $(\widetilde{\mathcal{P}})$ with associated state $\left(\widetilde{\mathbf{y}}^{1}, \widetilde{\mathbf{y}}^{2}\right) \in\left(W^{1,2,2}\left(0,1 ; \mathbf{V}_{1}, \mathbf{V}_{1}^{\prime}\right) \cap\left[L^{\infty}\left((0,1) \times \Omega_{1}\right)\right]^{4}\right) \times\left(W^{1,2,2}\left(0,1 ; \mathbf{V}_{2}, \mathbf{V}_{2}^{\prime}\right) \cap\right.$ $\left.\left[L^{\infty}\left((0,1) \times \Omega_{2}\right)\right]^{4}\right)$ such that $\widetilde{\mathbf{y}}^{j}(\tau)=\left(\widetilde{\mathbf{u}}^{j} \circ \widetilde{\phi}^{j}\right)(\tau), j=1,2$. Then, from the results of Theorem 4.7, there will exist elements $\gamma \geq 0, \lambda \in \mathbb{R}^{4}$, with $(\gamma, \lambda) \neq 0,\left(\mathbf{q}^{1}, \mathbf{q}^{2}\right) \in\left(W^{1,2,2}\left(0,1 ; \mathbf{V}_{1}, \mathbf{V}_{1}^{\prime}\right) \cap\left[L^{\infty}\left((0,1) \times \Omega_{1}\right)\right]^{4}\right) \times$ $\left(W^{1,2,2}\left(0,1 ; \mathbf{V}_{2}, \mathbf{V}_{2}^{\prime}\right) \cap\left[L^{\infty}\left((0,1) \times \Omega_{2}\right)\right]^{4}\right)$, and $\left(r^{1}, r^{2}\right) \in \mathcal{C}([0,1] ; \mathbb{R})^{2}$, satisfying the systems (4.45)-(4.47) and verifying the conditions (4.42)-(4.44).

Now, we will define the adjoint states associated to the distributed controls $\left(\mathbf{p}^{1}, \mathbf{p}^{2}\right) \in\left(W^{1,2,2}\left(0, \widetilde{T}^{1} ; \mathbf{V}_{1}, \mathbf{V}_{1}^{\prime}\right) \cap\right.$ $\left.\left[L^{\infty}\left(\left(0, \widetilde{T}^{1}\right) \times \Omega_{1}\right)\right]^{4}\right) \times\left(W^{1,2,2}\left(0, \widetilde{T}^{2} ; \mathbf{V}_{2}, \mathbf{V}_{2}^{\prime}\right) \cap\left[L^{\infty}\left(\left(0, \widetilde{T}^{2}\right) \times \Omega_{2}\right)\right]^{4}\right.$ associated to the distributed controls for the problem $(\mathcal{P})$, given by:

$$
\mathbf{q}^{j}(\tau)=\left(\mathbf{p}^{j} \circ \widetilde{\phi}^{j}\right)(\tau), \quad j=1,2,
$$

and define the adjoint states $\left(s^{1}, s^{2}\right) \in \mathcal{C}\left(\left[0, \widetilde{T}^{1}\right] ; \mathbb{R}\right) \times \mathcal{C}\left(\left[0, \widetilde{T}^{2}\right] ; \mathbb{R}\right)$ associated to the time controls for the problem $(\mathcal{P})$ as:

$$
r^{j}(\tau)=\left(s^{j} \circ \widetilde{\phi}^{j}\right)(\tau), \quad j=1,2 .
$$

It is clear, by using the changes of variables $t^{j}=\widetilde{\phi}^{j}(\tau), j=1,2$, that the pair $\left(\mathbf{p}^{1}, \mathbf{p}^{2}\right)$ is solution of the systems (4.59)-(4.60), and that the pair $\left(s^{1}, s^{2}\right)$ is solution of the problems (4.61), for $j=1,2$. These changes of variable also allows us to obtain, in a direct manner, that condition (4.42) is equivalent to condition (4.56), and that condition (4.43) leads to condition (4.57).

However, obtaining condition (4.58) is not so immediate from condition (4.44), so we will need to apply more refined arguments: Let us begin by analyzing the extreme case where any of the optimal times satisfies $\widetilde{T}^{j}=T_{\min }$. It is obvious in this case that the only possible choice of the mapping $\widetilde{v}^{j}$ associated to the change of variable will be $\widetilde{v}^{j}(\tau)=T_{\min }, \forall \tau \in[0,1]$. If we consider now an arbitrary mapping $h \in \mathcal{C}([0,1] ; \mathbb{R})$ such that $1 \leq h(\tau) \leq \frac{T_{\max }}{T_{\min }}, \forall \tau \in[0,1]$, it is evident that $v^{j}(\tau)=T_{\min } h(\tau)$ is a time control satisfying the constraints imposed for the definition of $\mathcal{W}_{a d}$. Thus, from condition (4.44), we have that:

$$
\begin{aligned}
& \int_{0}^{1}\left(v^{j}(\tau)-\widetilde{v}^{j}(\tau)\right)\left[r^{j}(\tau)+\mathcal{H}^{j}\left(\widetilde{\phi}^{j}(\tau), \widetilde{\mathbf{y}}^{j}(\tau), \mathbf{q}^{j}(\tau), \widetilde{\varrho}^{j}(\tau), \gamma\right)\right] \mathrm{d} \tau=\int_{0}^{1} T_{\min }(h(\tau)-1)\left[r^{j}(\tau)\right. \\
& \left.+\mathcal{H}^{j}\left(\widetilde{\phi}^{j}(\tau), \widetilde{\mathbf{y}}^{j}(\tau), \mathbf{q}^{j}(\tau), \widetilde{\varrho}^{j}(\tau), \gamma\right)\right] \mathrm{d} \tau=\int_{0}^{T_{\min }}\left(h \circ\left(\widetilde{\phi}^{j}\right)^{-1}\left(t^{j}\right)-1\right)\left[s^{j}\left(t^{j}\right)-\mathcal{H}^{j}\left(t^{j}, \widetilde{\mathbf{u}}^{j}\left(t^{j}\right), \mathbf{p}^{j}\left(t^{j}\right), \widetilde{\rho}^{j}\left(t^{j}\right), \gamma\right)\right] \mathrm{d} t^{j} \geq 0,
\end{aligned}
$$


TABle 1. Coefficients for numerical example.

\begin{tabular}{|c|c|c|}
\hline Coefficients & Values & Units \\
\hline$\mu_{i}, i=1, \ldots, 4$ & $2.00 \times 10^{-3}$ & $\mathrm{~m}^{2} \cdot \mathrm{s}^{-1}$ \\
$C_{n c}$ & $2.70 \times 10^{-1}$ & - \\
$K_{N}$ & $2.80 \times 10^{-2}$ & $\mathrm{mg} \cdot \mathrm{L}^{-1}$ \\
$K_{F}$ & $2.00 \times 10^{-1}$ & $\mathrm{mg} \cdot \mathrm{L}^{-1}$ \\
$K_{m f}$ & $3.80 \times 10^{-7}$ & $\mathrm{~s}^{-1}$ \\
$K_{m z}$ & $3.78 \times 10^{-7}$ & $\mathrm{~s}^{-1}$ \\
$K_{r}$ & $3.80 \times 10^{-7}$ & $\mathrm{~s}^{-1}$ \\
$K_{z}$ & $2.30 \times 10^{-6}$ & $\mathrm{~s}^{-1}$ \\
$K_{r d}$ & $2.30 \times 10^{-5}$ & $\mathrm{~s}^{-1}$ \\
$C_{f z}$ & $6.00 \times 10^{-1}$ & - \\
$\Theta$ & 1.05 & - \\
$\theta$ & 19.00 & $\circ \mathrm{C}^{-1}$ \\
$\nu$ & $1.50 \times 10^{-5}$ & $\mathrm{~s}^{-1}$ \\
$\phi$ & $8.50 \times 10^{-1}$ & $\mathrm{~m}^{-1}$ \\
$I_{0}$ & 100.00 & $\mathrm{Cal} \cdot \mathrm{m}^{-2} \cdot \mathrm{s}^{-1}$ \\
$I_{s}$ & 90.00 & $\mathrm{Cal} \cdot \mathrm{m}^{-2} \cdot \mathrm{s}$ \\
$C_{t}$ & 1.06 & - \\
$W_{f d}$ & $1.00 \times 10^{-2}$ & $\mathrm{~m} \cdot \mathrm{s}^{-1}$ \\
\hline
\end{tabular}

from which we can deduce that:

$$
s^{j}\left(t^{j}\right)-\mathcal{H}^{j}\left(t^{j}, \widetilde{\mathbf{u}}^{j}\left(t^{j}\right), \mathbf{p}^{j}\left(t^{j}\right), \widetilde{\rho}^{j}\left(t^{j}\right), \gamma\right) \geq 0 \quad \text { a.e. } t^{j} \in\left[0, T_{\min }\right]
$$

For the other extreme case $\widetilde{T}^{j}=T_{\max }$, we have that $\widetilde{v}^{j}(\tau)=T_{\max }, \forall \tau \in[0,1]$, and, arguing as in previous case, we obtain that:

$$
s^{j}\left(t^{j}\right)-\mathcal{H}^{j}\left(t^{j}, \widetilde{\mathbf{u}}^{j}\left(t^{j}\right), \mathbf{p}^{j}\left(t^{j}\right), \widetilde{\rho}^{j}\left(t^{j}\right), \gamma\right) \leq 0 \quad \text { a.e. } \quad t^{j} \in\left[0, T_{\max }\right] .
$$

Finally, for the intermediate case $T_{\min }<\widetilde{T}^{j}<T_{\max }$, we also have that:

$$
s^{j}\left(t^{j}\right)-\mathcal{H}^{j}\left(t^{j}, \widetilde{\mathbf{u}}^{j}\left(t^{j}\right), \mathbf{p}^{j}\left(t^{j}\right), \widetilde{\rho}^{j}\left(t^{j}\right), \gamma\right)=0 \quad \text { a.e. } t^{j} \in\left[0, \widetilde{T}^{j}\right]
$$

From any of the above three conditions (4.62)-(4.64) we can obtain condition (4.58), which concludes the proof.

We must note here that the choice of the time controls in space $\mathcal{C}\left([0,1] ; \mathbb{R}_{*}^{+}\right)$have allowed us to obtain condition (4.58). However, if we had chosen the time controls simply in $\mathbb{R}_{*}^{+}$(corresponding to linear - and simpler - changes of variable of the type $t=\phi(\tau)=T \tau$, with $T>0$ ), we would obtain, instead of optimality condition (4.58), the more restrictive condition:

$$
\sum_{j=1}^{2}\left(T^{j}-\widetilde{T}^{j}\right) \int_{0}^{\widetilde{T}^{j}}\left[s^{j}(t)-\mathcal{H}^{j}\left(t, \widetilde{\mathbf{u}}^{j}(t), \mathbf{p}^{j}(t), \widetilde{\rho}^{j}(t), \gamma\right)\right] \mathrm{d} t \geq 0, \quad \forall\left(T^{1}, T^{2}\right) \in \mathcal{V}_{a d}^{1}
$$

It is also worthwhile remarking here that, for the particular case when the water velocities are null (that is, $\mathbf{w}^{j}=0$, for $j=1,2$ ) and the water temperatures are smooth enough, similar optimality conditions have been obtained (see, for instance, Raymond and Zidani $[15,16]$ ) by using Pontryagin's maximum principle. 
TABLE 2. Results for numerical example.

\begin{tabular}{|c|c|c|}
\hline Element & Initial value & Optimal value \\
\hline$J$ & $4.86007 \times 10^{5}$ & $1.57356 \times 10^{5}$ \\
$B^{1}$ & $2.22887 \times 10^{-1}$ & $2.19282 \times 10^{-1}$ \\
$B^{2}$ & $2.20001 \times 10^{-1}$ & $1.80000 \times 10^{-1}$ \\
$B^{3}$ & $3.98949 \times 10^{-2}$ & $2.05899 \times 10^{-2}$ \\
$B^{4}$ & $5.67390 \times 10^{-2}$ & $3.99999 \times 10^{-2}$ \\
\hline
\end{tabular}

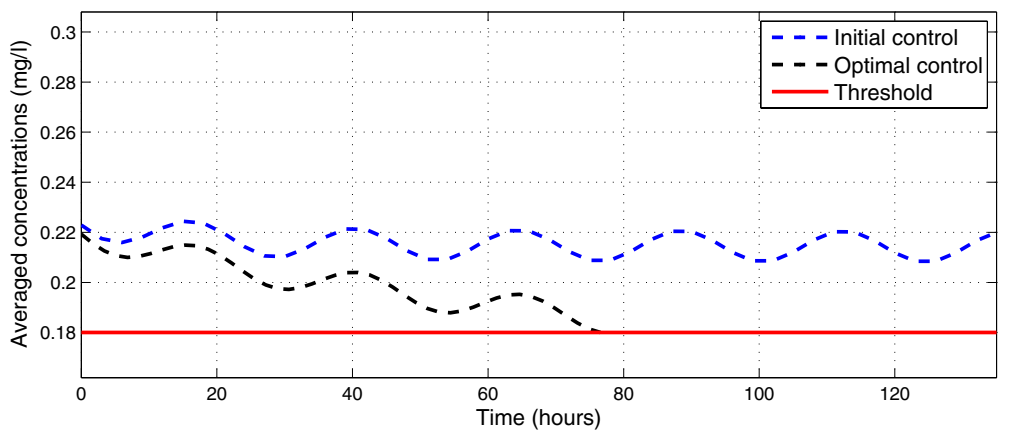

Figure 1. Averaged concentrations of nitrogen in the second tank $\Omega_{2}$.

\section{Practical conclusions}

After a long (and sometimes tedious) derivation, we obtain existence of optimal solutions for the control problem and a first order optimality condition in order to characterize them. This optimality condition can be used - after a discretization process - to compute a numerical solution of the original problem. For the sake of completeness, we introduce here some numerical results obtained for a realistic example consisting of two tanks of equal dimensions $20 \mathrm{~m} \times 20 \mathrm{~m} \times 16 \mathrm{~m}$. We consider the physical coefficients as given in Table 1 .

Moreover, the initial conditions for the first tank will be given by $u_{0}^{1,1}=0.28 \mathrm{mg} \cdot \mathrm{L}^{-1}, u_{0}^{2,1}=0.01 \mathrm{mg} \cdot \mathrm{L}^{-1}$, $u_{0}^{3,1}=0.02 \mathrm{mg} \cdot \mathrm{L}^{-1}$ and $u_{0}^{4,1}=4.50 \mathrm{mg} \cdot \mathrm{L}^{-1}$.

For the other values related to state and control constraints, we have taken the thresholds $\sigma_{1}=0.23$, $\sigma_{2}=0.18, \theta_{1}=0.015$ and $\theta_{2}=0.04$ (i.e. we are imposing a nitrogen reduction of the $82 \%$ in the first tank and of the $64 \%$ in the second one), a phytoplankton upper bound $K=100$, and time bounds $T_{\min }=135 / 2=67.5$ and $T_{\max }=2 \times 135=270$, i.e., we are restricting our search to time variations between the half and the double of a starting time period of $135 \mathrm{~h}$.

Finally, for the numerical solution of the eutrophication systems, we have considered a tetrahedral regular mesh of both tanks formed by $P_{1}$ finite elements with a characteristic size of $1 \mathrm{~m}$, and - for a starting time period of $135 \mathrm{~h}$ - a time step length of $3 \mathrm{~h}$ (with a total number of 45 time steps).

Then, applying an interior-point algorithm we have passed, in 22 iterations, from the initial times $T^{1}=$ $T^{2}=135$, to the optimal times $T^{1}=77.05$ and $T^{2}=76.57$. Thus, as a first consequence, we have obtained a significant time reduction for the whole process of about a $42 \%$. The values showing the reduction of the objective function and the accomplishment of the state constraints can be seen in Table 2 .

In Figure 2 we can see the averaged concentrations of organic detritus for the second tank, corresponding to the initial and the optimal controls. We can observe how, after the optimal permanence time $T^{2}$, the fourth constraint $B^{4}$ is also active.

In Figure 1 we show the averaged concentrations of nitrogen for the second tank, corresponding to the initial and the optimal controls. We can observe how, after the optimal permanence time $T^{2}=76.57$, the second 


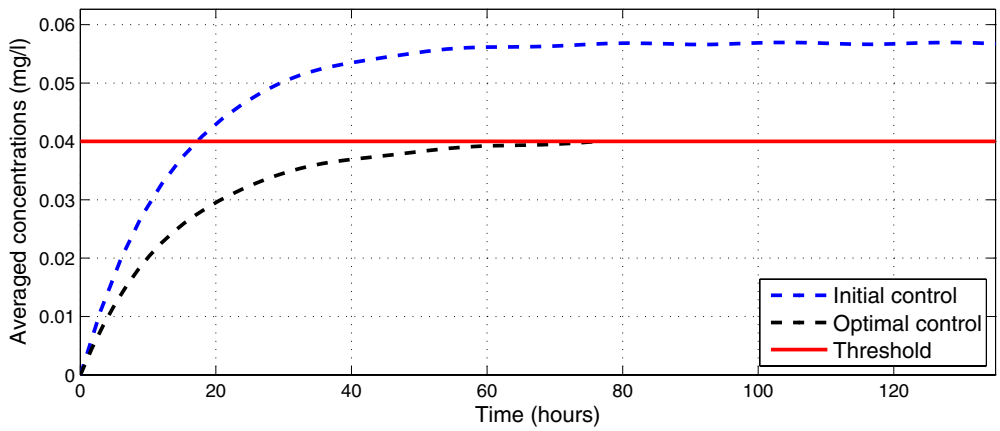

FIgURE 2. Averaged concentrations of organic detritus in the second tank $\Omega_{2}$.

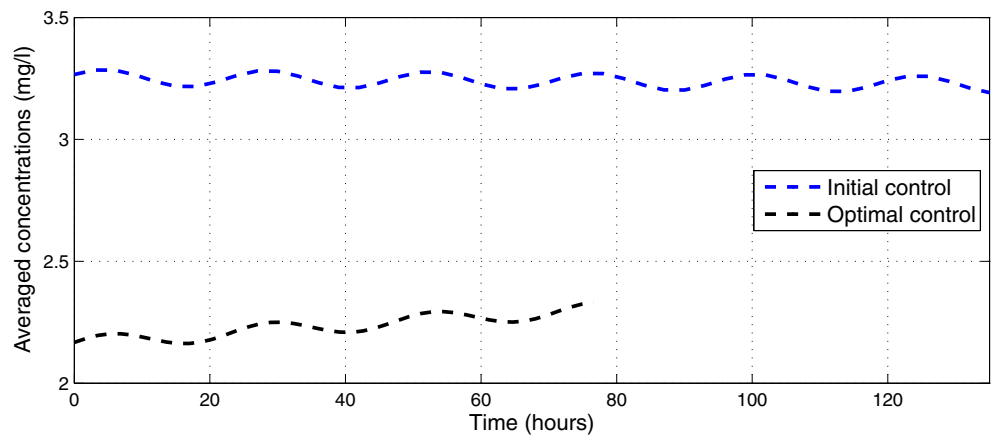

FIGURE 3. Averaged concentrations of phytoplankton in the second tank $\Omega_{2}$.

constraint $B^{2}$ reaches the exact threshold $\sigma_{2}$. Moreover, daily oscillations due to night/day luminosity variations can be clearly identified.

Finally, in Figure 3 we show the averaged concentrations of phytoplankton for the second tank, corresponding to the initial and the optimal controls. We can see the significant decrease of the value obtained for the controlled case, which stays always under the value obtained for the uncontrolled one.

\section{REFERENCES}

[1] W. Allegretto, C. Mocenni and A. Vicino, Periodic solutions in modelling lagoon ecological interactions. J. Math. Biol. 51 (2005) 367-388.

[2] L.J. Alvarez-Vázquez, F.J. Fernández and R. Muñoz-Sola, Analysis of a multistate control problem related to food technology. J. Differ. Equ. 245 (2008) 130-153.

[3] L.J. Alvarez-Vázquez, F.J. Fernández and R. Muñoz-Sola, Mathematical analysis of a three-dimensional eutrophication model. J. Math. Anal. Appl. 349 (2009) 135-155.

[4] N. Arada and J.-P. Raymond, Time optimal problems with Dirichlet boundary controls. Discrete Contin. Dyn. Syst. 9 (2003) $1549-1570$.

[5] O. Arino, K. Boushaba and A. Boussouar, A mathematical model of the dynamics of the phytoplankton-nutrient system. Nonlinear Anal. Real World Appl. 1 (2000) 69-87.

[6] R.P. Canale, Modeling biochemical processes in aquatic ecosystems. Ann Arbor Science Publishers, Ann Arbor (1976).

[7] P. Cannarsa and H. Frankowska, Interior sphere property of attainable sets and time optimal control problems. ESAIM: COCV 12 (2006) 350-370

[8] E. Casas, Boundary control of semilinear elliptic equations with pointwise state constraints. SIAM J. Control Optim. 31 (1993) 993-1006. 
[9] F. Cioffi and F. Gallerano, Management strategies for the control of eutrophication processes in Fogliano lagoon (Italy): a long-term analysis using a mathematical model. Appl. Math. Model. 25 (2001) 385-426.

[10] M. Drago, B. Cescon and L. Iovenitti, A three-dimensional numerical model for eutrophication and pollutant transport. Ecol. Model. 145 (2001) 17-34.

[11] M. Gugat and G. Leugering, $L^{\infty}$-norm minimal control of the wave equation: on the weakness of the bang-bang principle. ESAIM: COCV 14 (2008) 254-283.

[12] S. Li and G. Wang, The time optimal control of the Boussinesq equations. Numer. Funct. Anal. Optim. 24 (2003) 163-180.

[13] F. Lunardini and G. Di Cola, Oxygen dynamics in coastal and lagoon ecosystems. Math. Comput. Model. 31 (2000) $135-141$.

[14] K. Park, H.-S. Jung, H.-S. Kim and S.-M. Ahn, Three-dimensional hydrodynamic-eutrophication model (HEM-3D): application to Kwang-Yang Bay, Korea. Mar. Environ. Res. 60 (2005) 171-193.

[15] J.P. Raymond and H. Zidani, Pontryagin's principle for time-optimal problems. J. Optim. Theory Appl. 101 (1999) 375-402.

[16] J.P. Raymond and H. Zidani, Time optimal problems with boundary controls. Differ. Integr. Equat. 13 (2000) $1039-1072$.

[17] T. Roubíček, Nonlinear partial differential equations with applications. Birkhäuser-Verlag, Basel (2005).

[18] G. Wang, The existence of time optimal control of semilinear parabolic equations. Syst. Control Lett. 53 (2004) $171-175$.

[19] L. Wang and G. Wang, The optimal time control of a phase-field system. SIAM J. Control Optim. 42 (2003) $1483-1508$.

[20] Y. Yamashiki, M. Matsumoto, T. Tezuka, S. Matsui and M. Kumagai, Three-dimensional eutrophication model for Lake Biwa and its application to the framework design of transferable discharge permits. Hydrol. Proc. 17 (2003) $2957-2973$.

[21] E. Zeidler, Nonlinear Functional Analysis and Its Applications - Part 3: Variational Methods and Optimization. SpringerVerlag, Berlin (1985). 